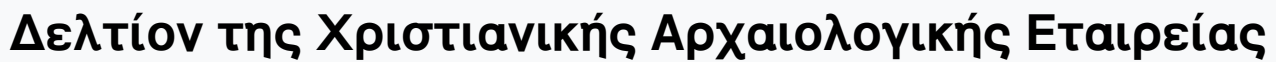

Tó 17 (1994)

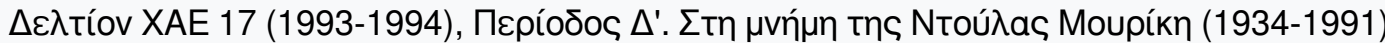

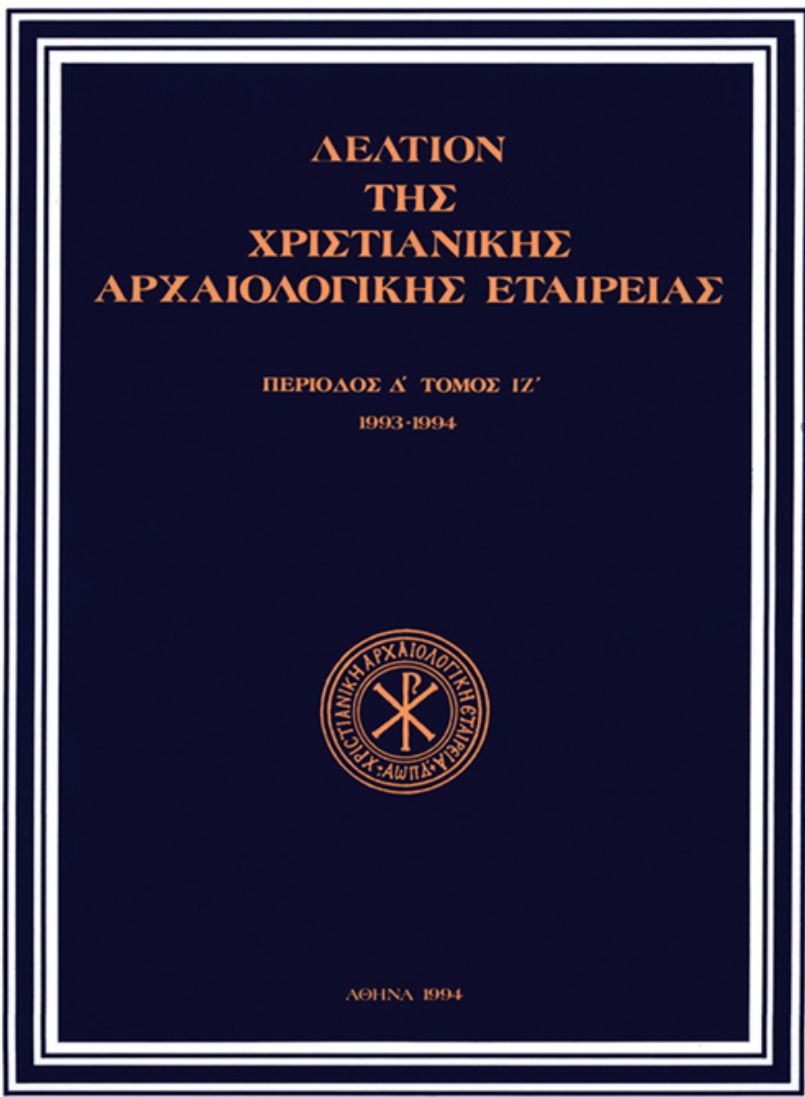

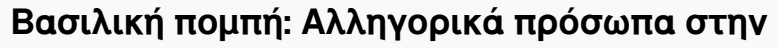
Aváotaon tns Dečani

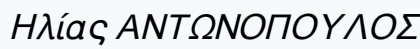

doi: $10.12681 /$ dchae.1094

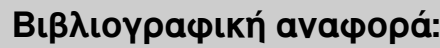

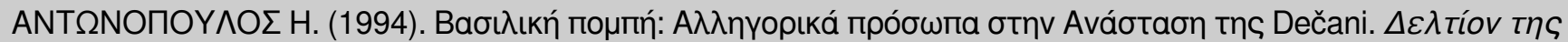

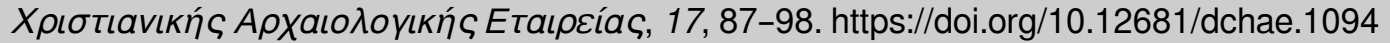




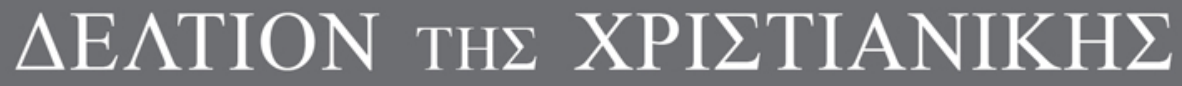 APXAIO $\Lambda$ OГIKH $\Sigma$ ETAIPEIA $\Sigma$}

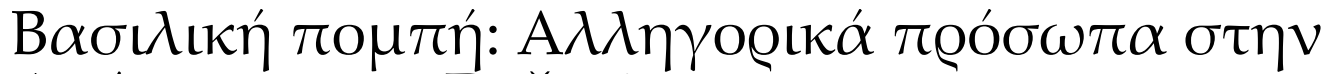

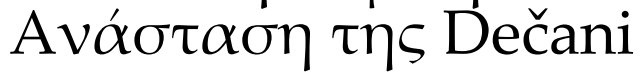

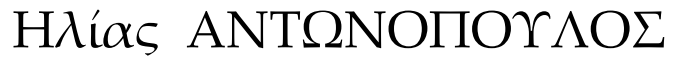

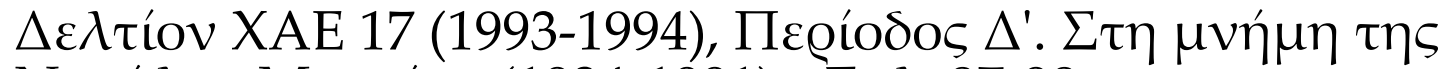

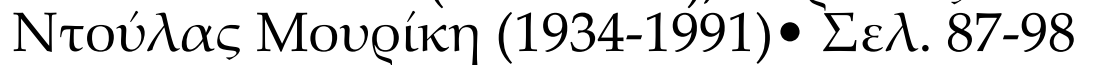

A@HNA 1994 


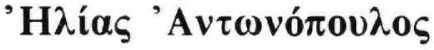

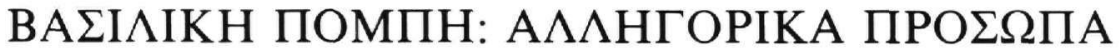 $\Sigma$ THN ANA $\Sigma$ TA $\Sigma$ H TH $\Sigma$ DEČANI*}

$\sum_{\tau}$

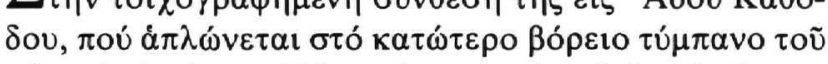

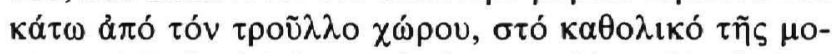

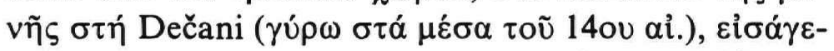

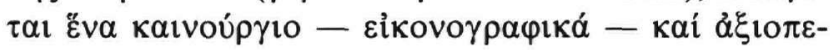

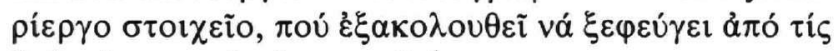

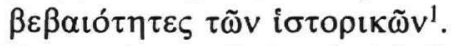

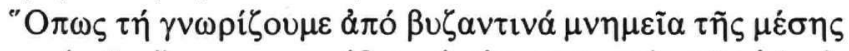

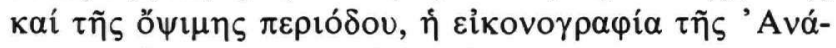

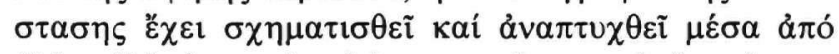

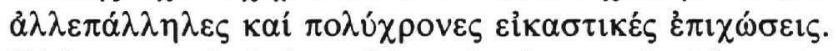

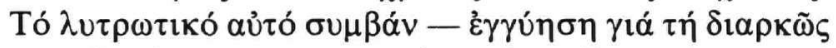

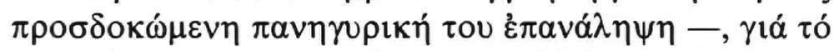

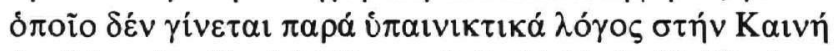

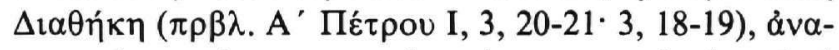

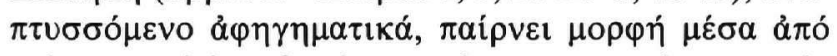

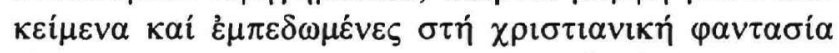

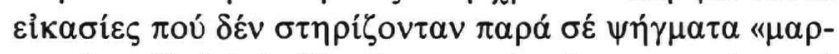

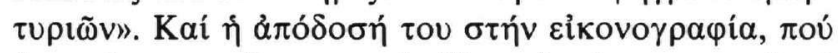

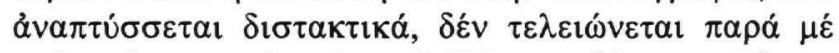

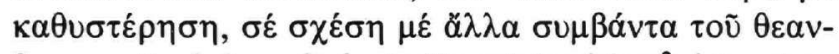

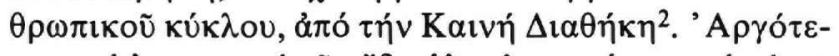

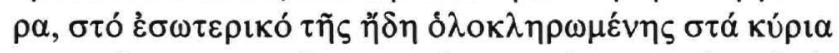

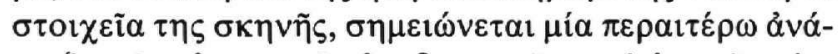

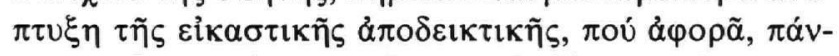

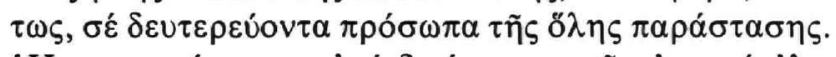

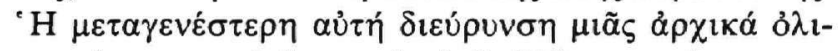

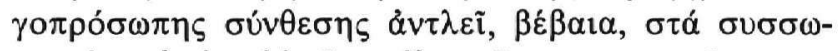

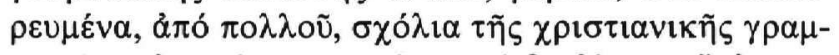

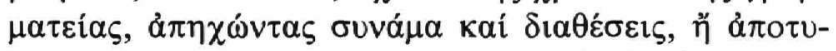

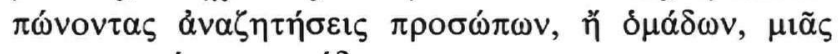

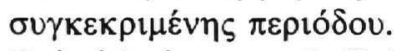

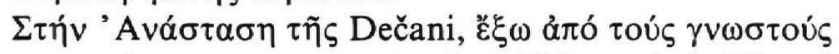

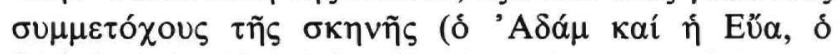

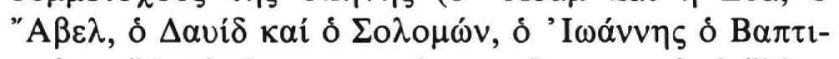

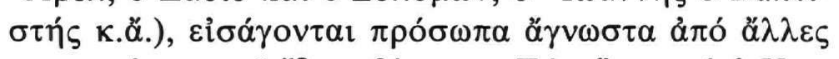

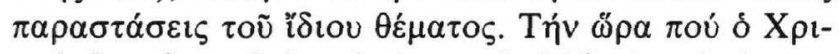

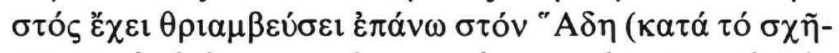

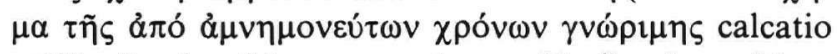

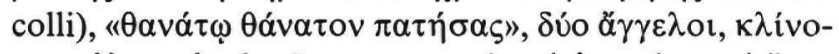

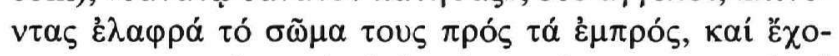

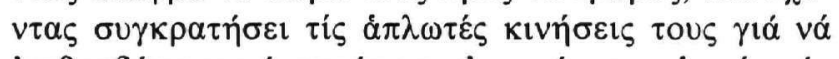

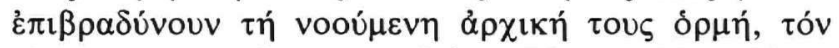

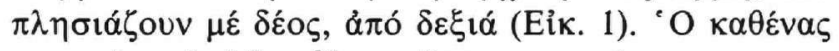

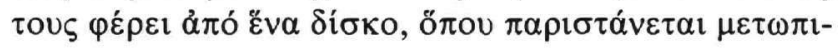

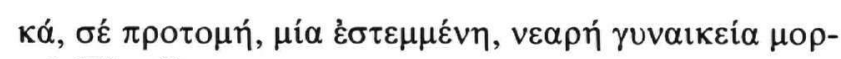

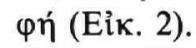

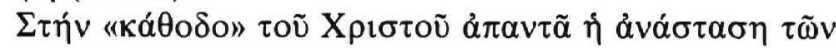

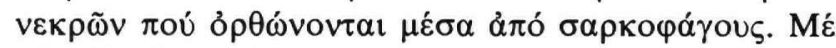

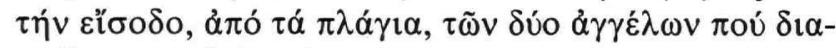

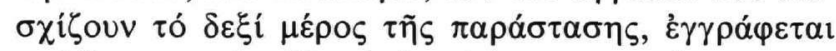

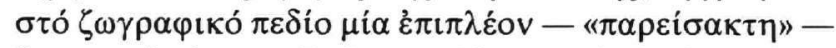

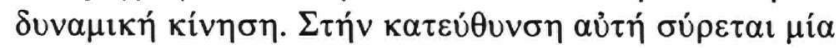

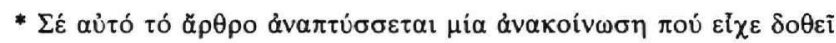

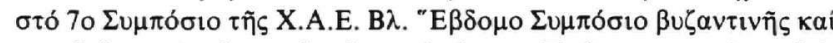

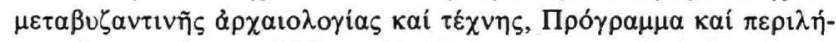

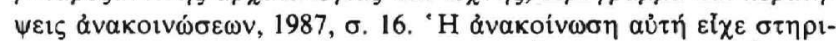

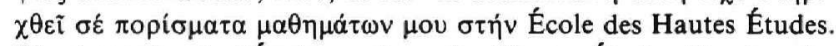
$\mathrm{B} \lambda$. Annuaire de l'École pratique des Hautes Études (Section des sciences religieuses), Résumés des conférences et travaux, 94 (1986), $\sigma$.

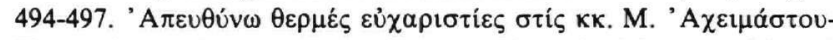

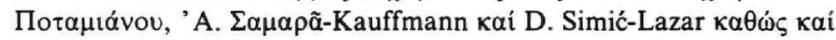

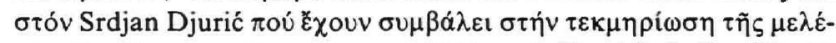

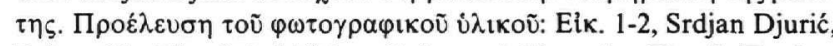

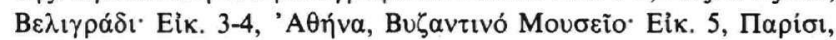

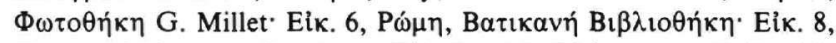

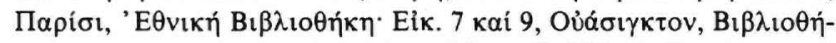

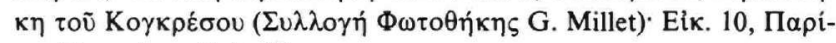

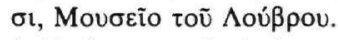

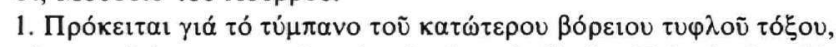

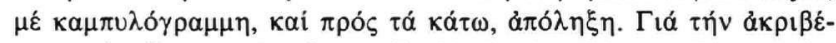

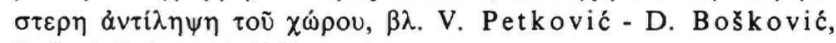

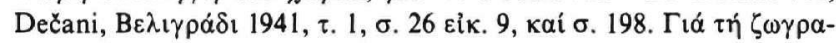

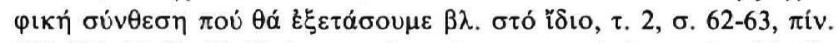
179-180. П $\rho \beta$. T. Velmans, La peinture murale byzantine à la fin

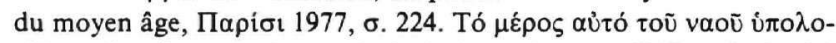

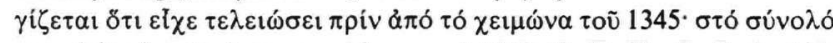

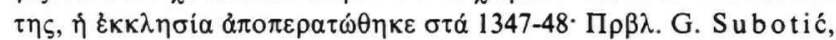
Contribution à la chronologie de la peinture murale de Dečani, Re-

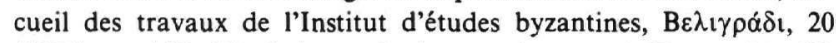

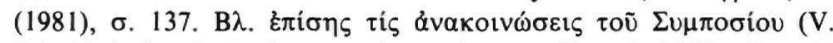
Djurić, ék $\delta$.): Dečani et l'art byzantin au milieu du XIVe siècle (à l'occasion de la célébration de 650 ans du monastère de Dečani, sept.

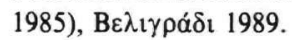

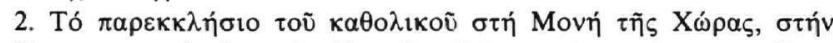

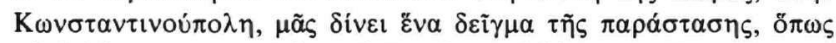

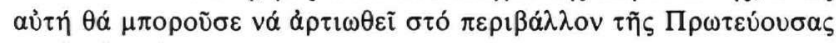

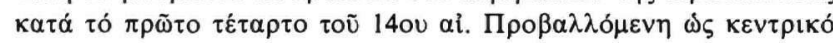

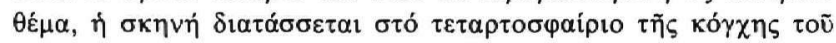

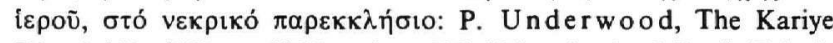

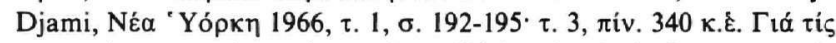

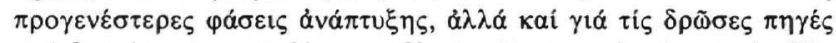

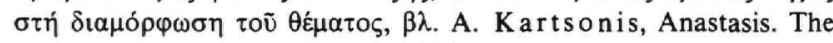
Making of an Image, Princeton 1986. 


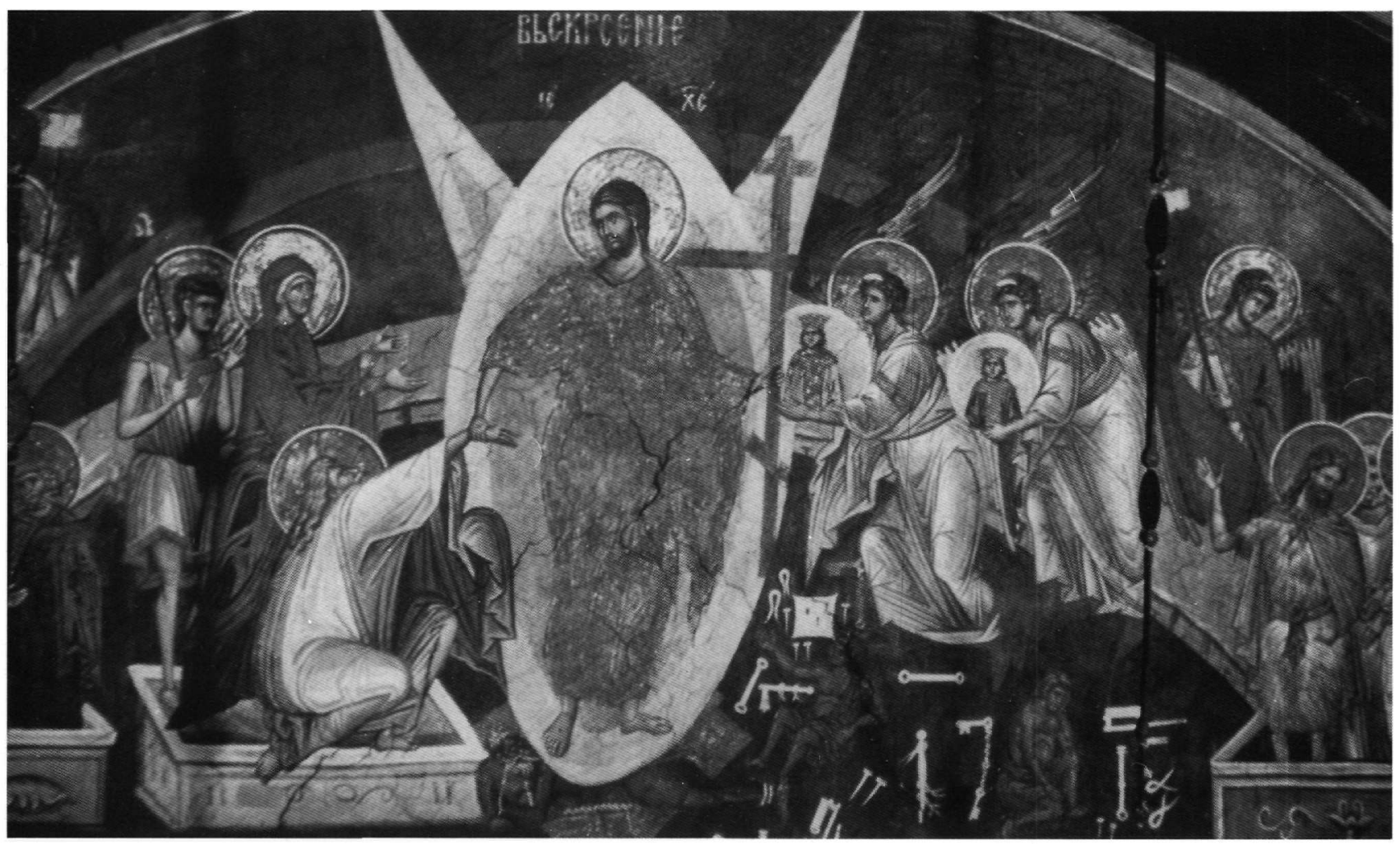

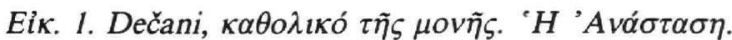

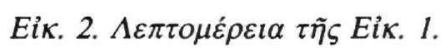

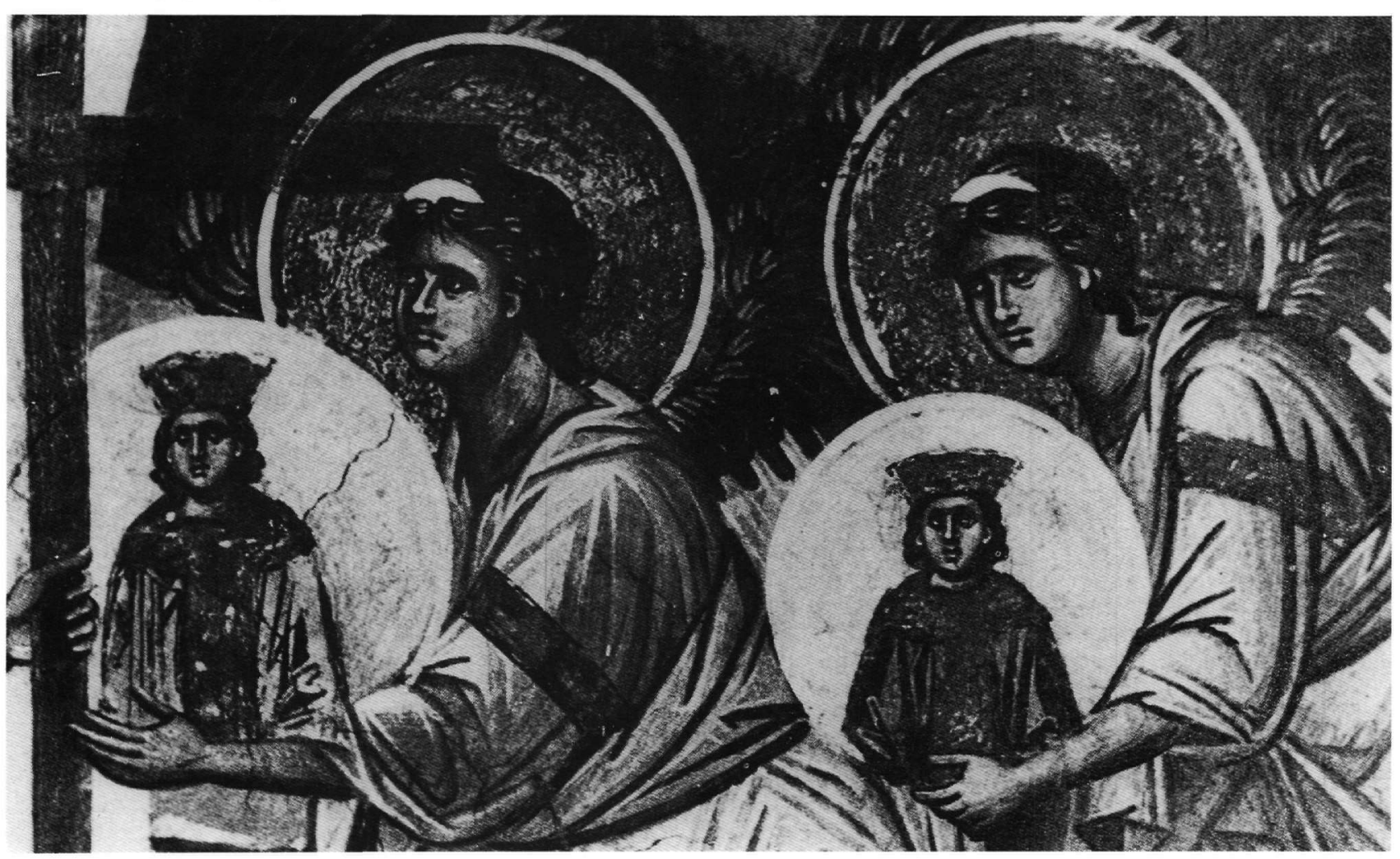




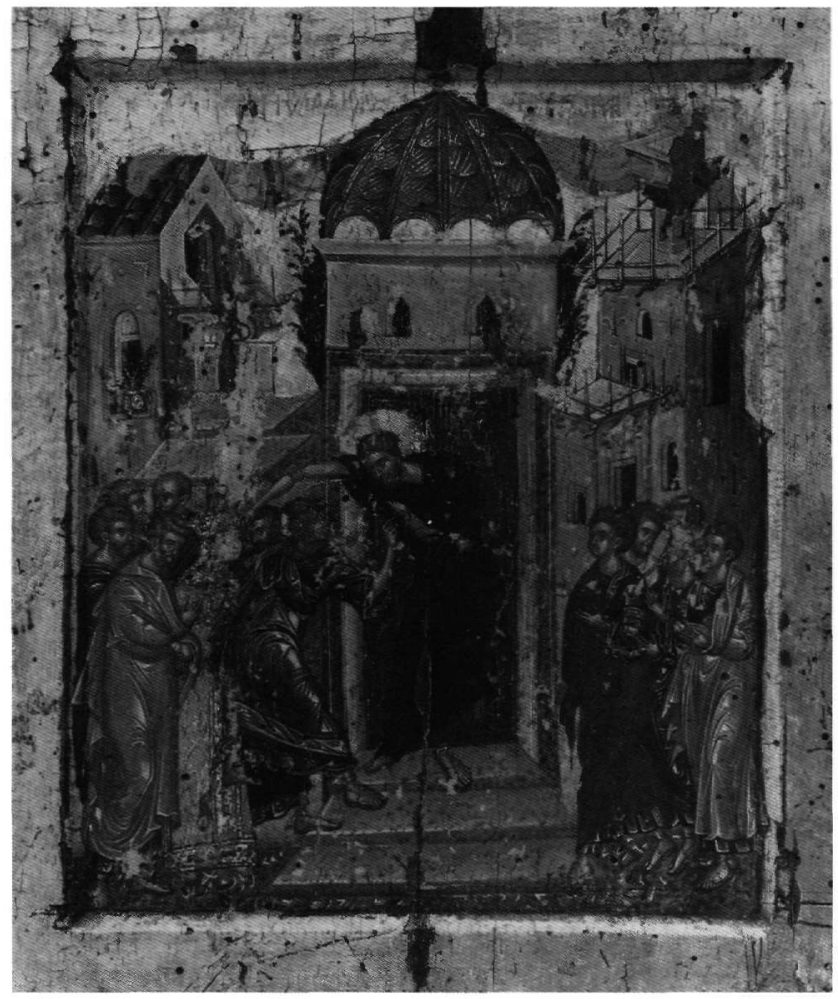

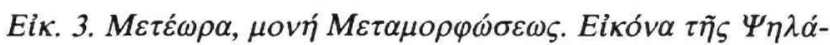
$\varphi \eta \sigma \eta \varsigma \tau \sigma \tilde{v} \Theta \omega \mu \tilde{\alpha}$.

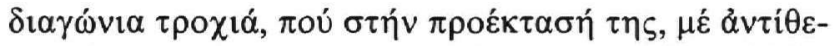

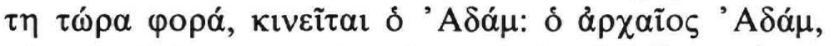

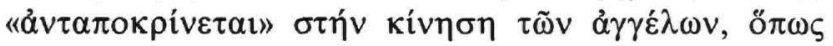

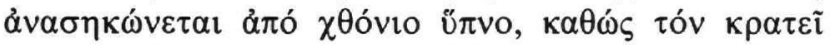

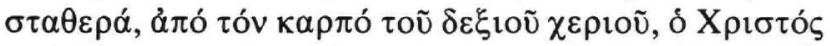

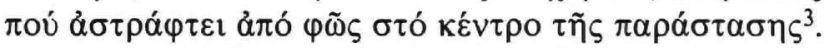

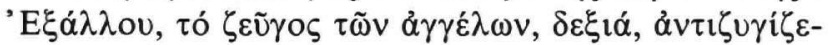

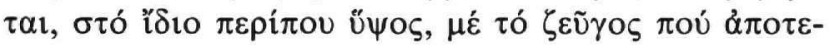

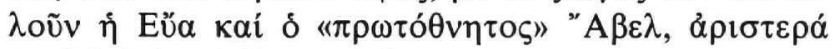

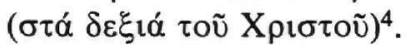

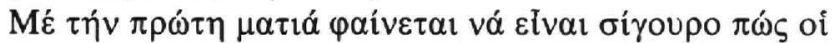

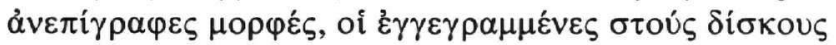

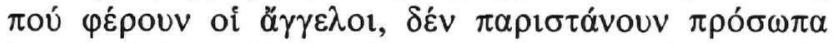

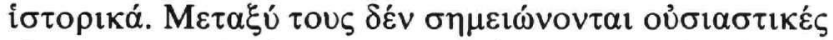

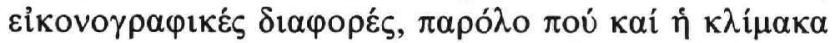

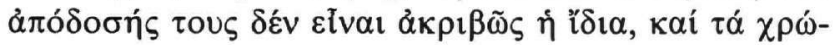

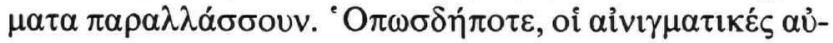

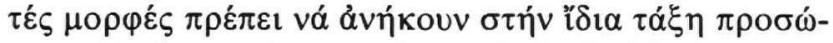

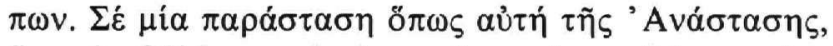

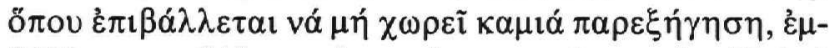

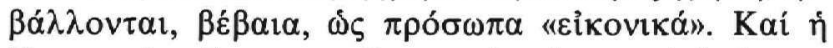

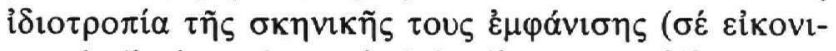

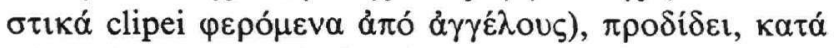

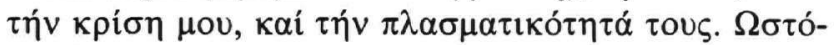

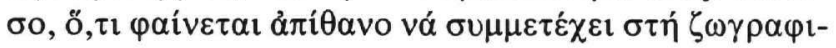

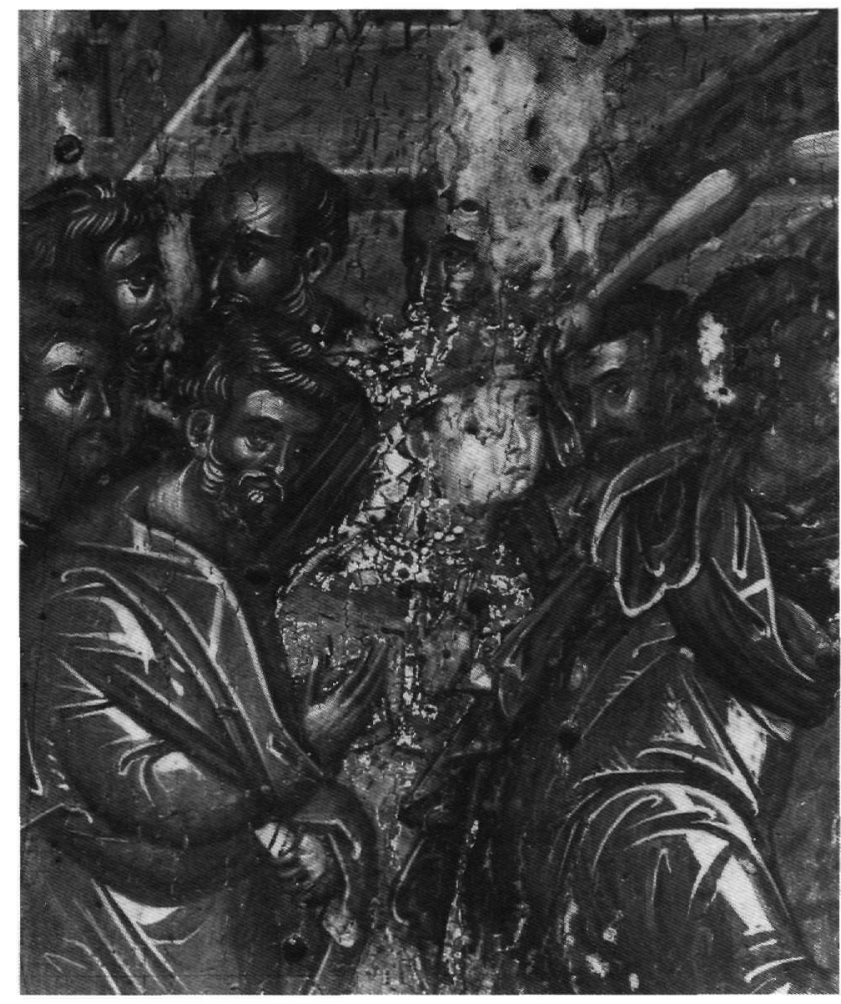

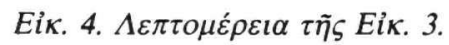

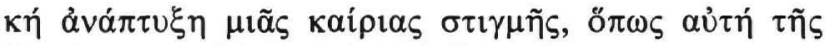

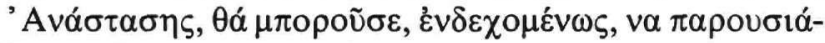

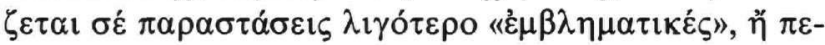

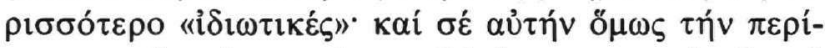

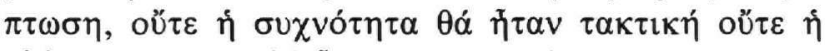

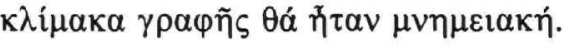

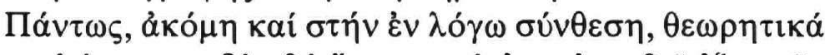

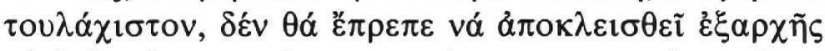

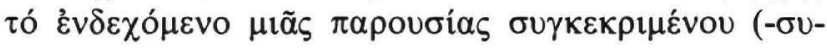

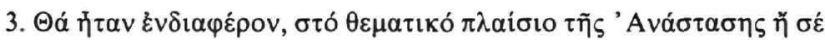

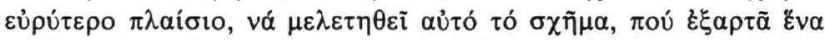

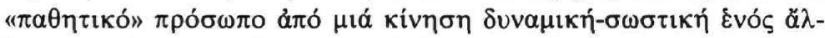

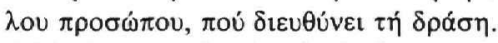

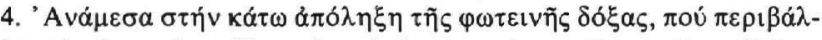

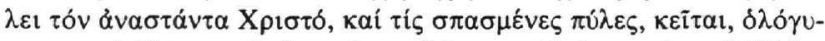

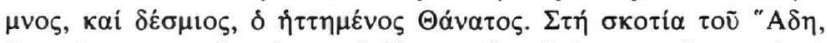

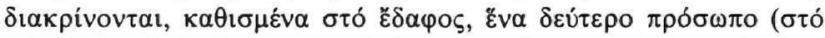

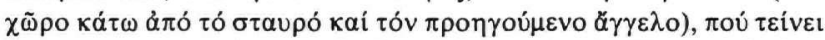

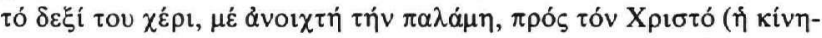
$\sigma \eta \delta \eta \lambda \dot{\omega} v \varepsilon 1 \mu \tilde{\alpha} \lambda \lambda$ ov $\alpha \alpha \tau \alpha \dot{\pi} \lambda \eta \xi \eta \eta$

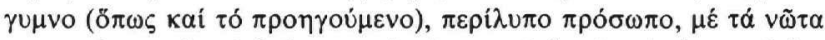

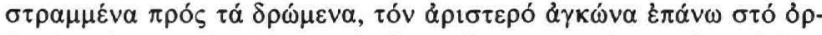

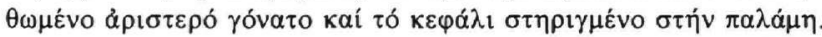

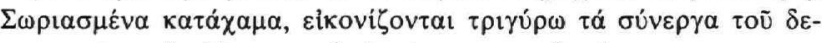

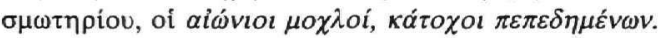




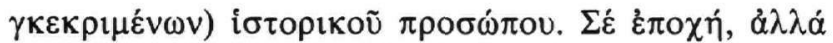

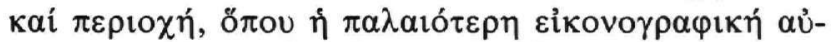

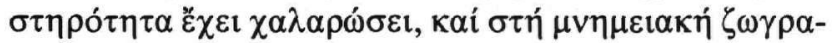

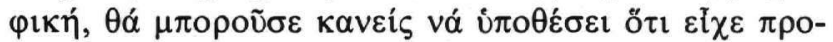

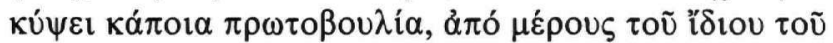

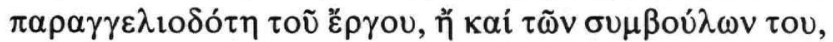

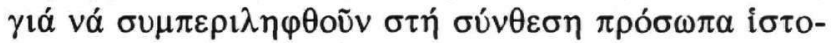

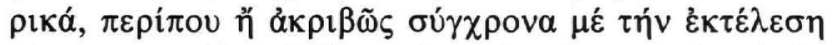

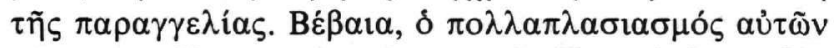

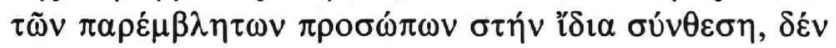

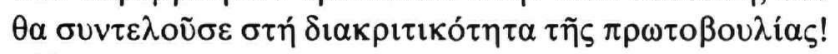

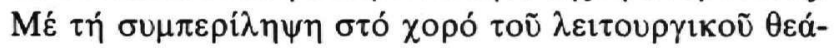

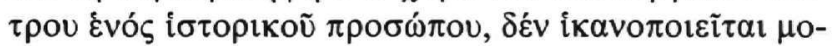

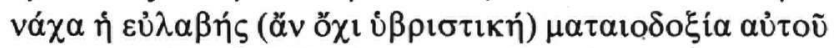

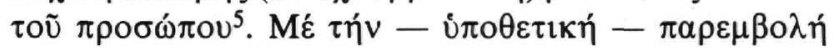

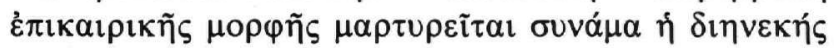

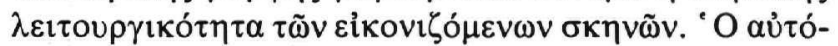

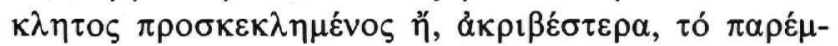

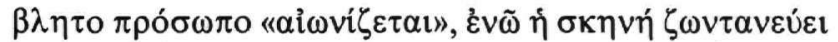

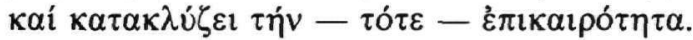

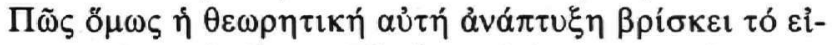

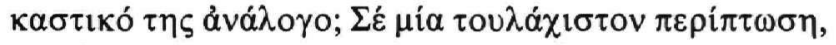

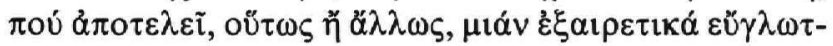

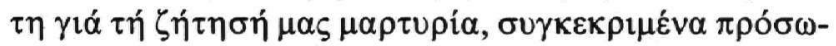

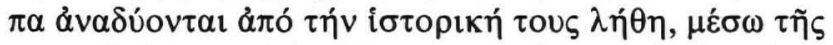

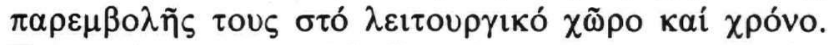

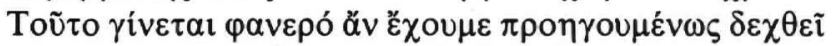

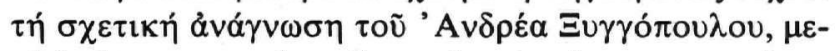

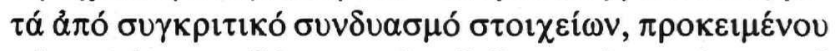

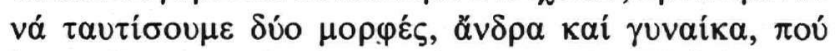

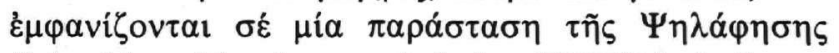

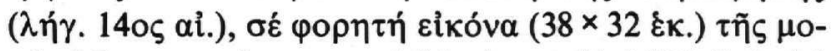

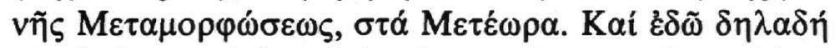

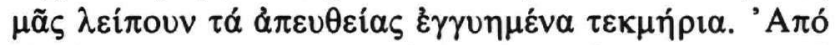

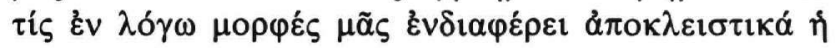

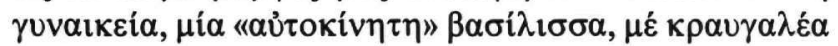

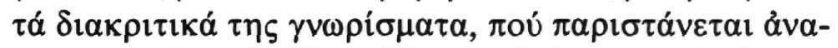

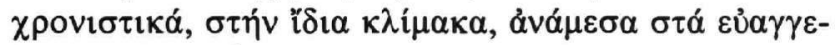
$\lambda \iota \kappa \alpha ́$ $\pi \rho \sigma_{\sigma} \omega \pi \alpha^{6}$.

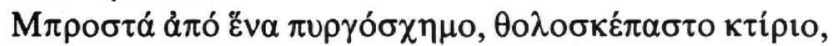

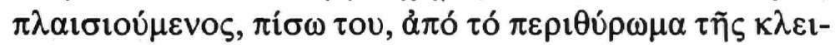

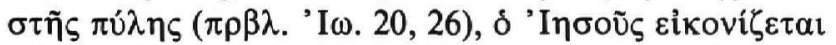

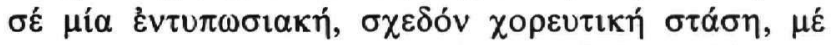

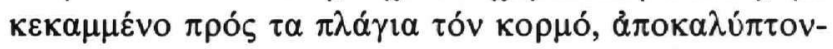

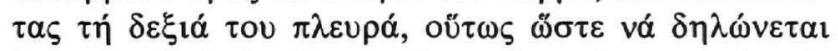

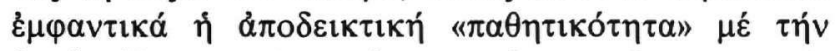

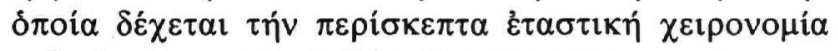

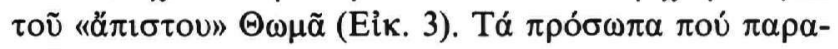

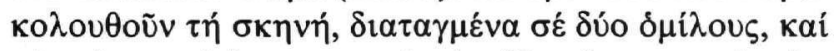

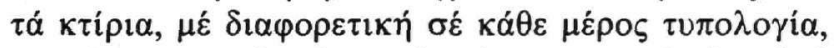

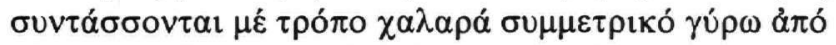

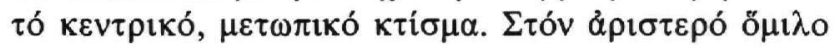

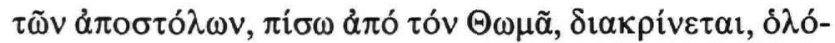

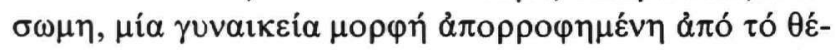

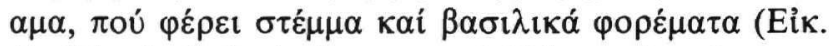

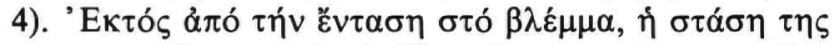

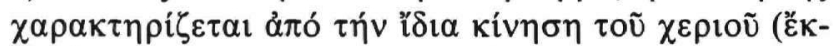

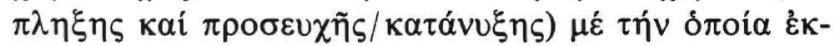

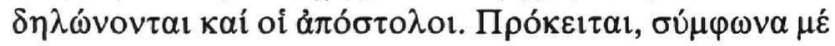

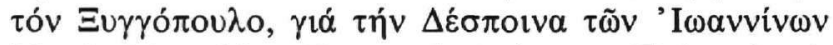

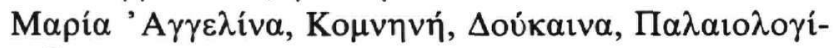
$v \alpha^{7}$.

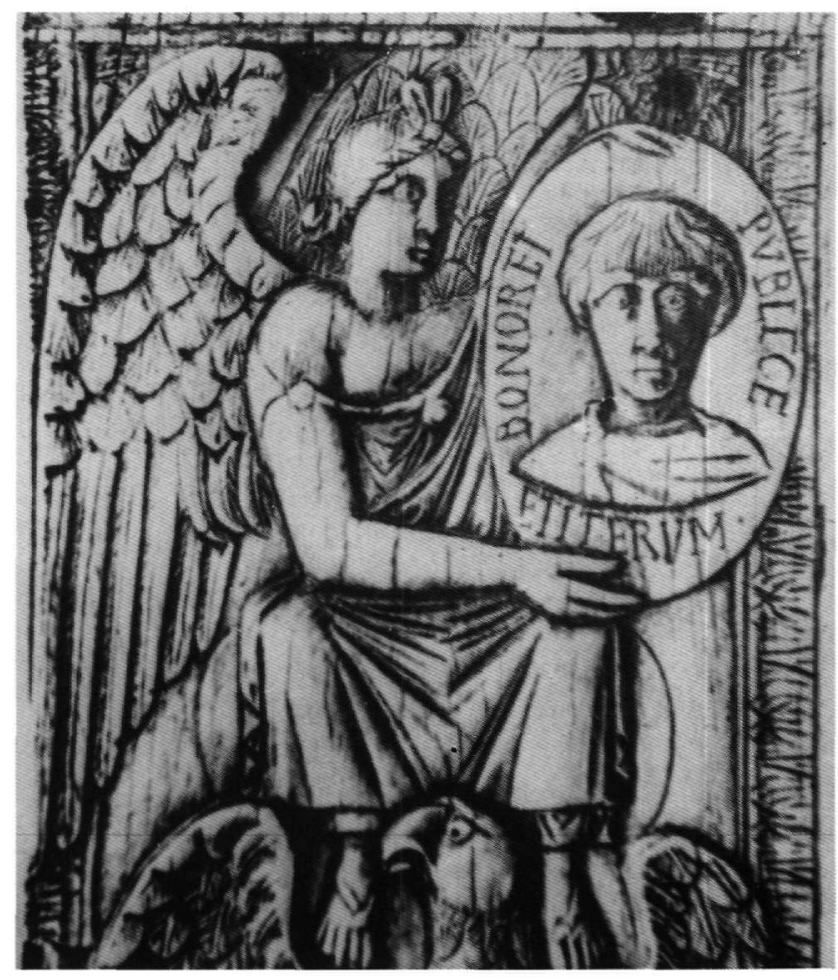

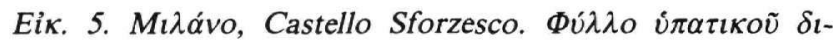
$\pi \tau \dot{\chi} \chi 0 \mathrm{~s}$

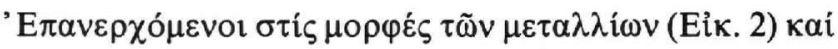

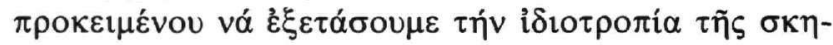

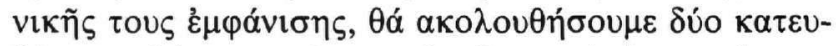

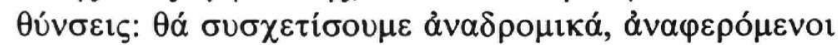

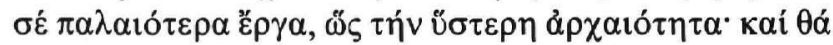

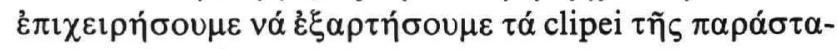

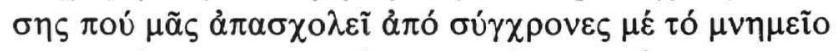

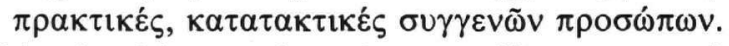

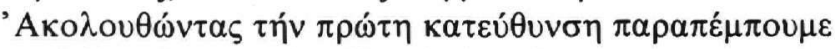

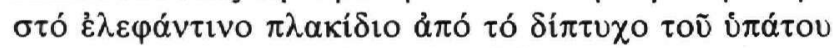

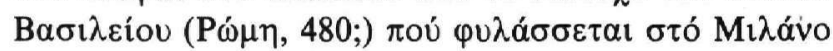

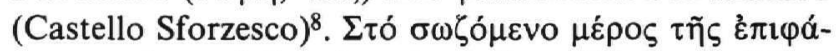

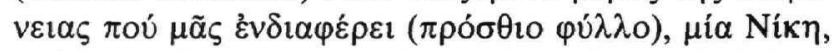

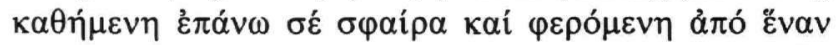

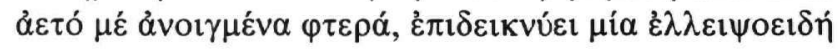




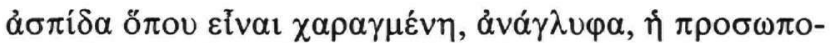

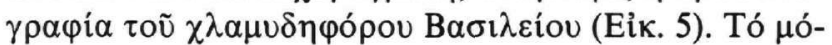

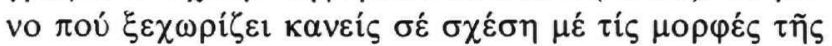

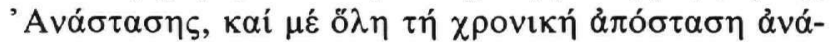

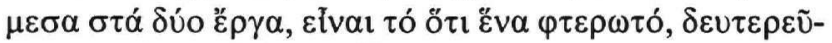

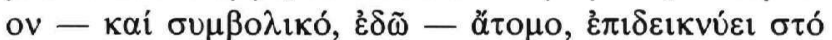

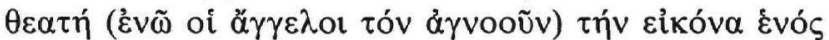

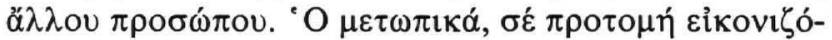

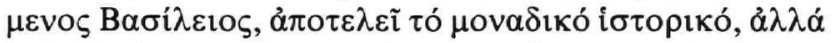

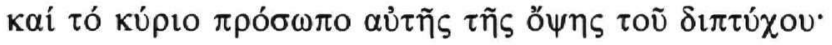

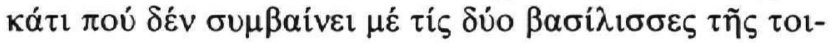

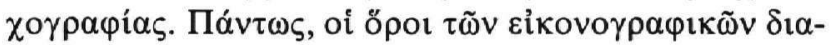

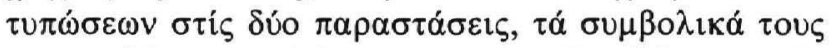

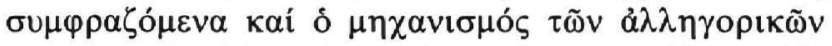

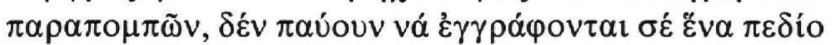

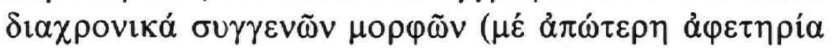

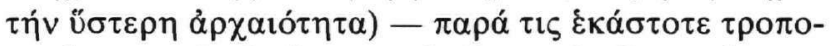

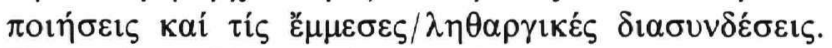

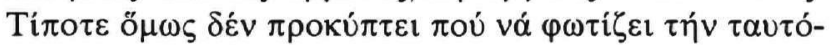

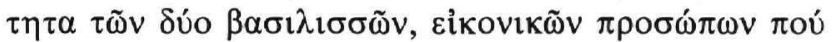

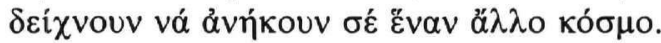

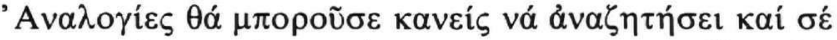

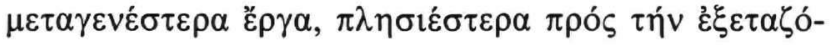
$\mu \varepsilon v \eta \pi \alpha \rho \alpha ́ \sigma \tau \alpha \sigma \eta, \mu \varepsilon \dot{\varepsilon} \mu \imath \gamma \tilde{\omega} \varsigma \chi \rho \imath \tau \tau \imath \alpha v i \kappa \eta ́ ~ \varepsilon i ̉ \kappa o v o \gamma \rho \alpha-$

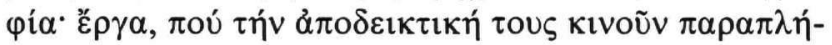

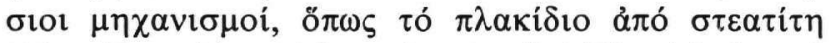

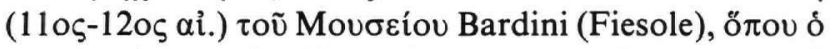

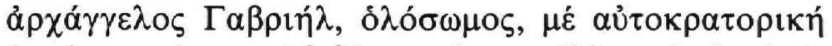

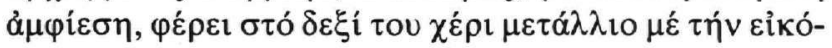

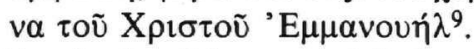

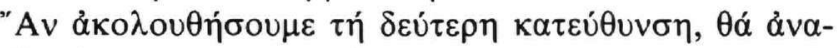

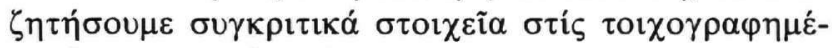

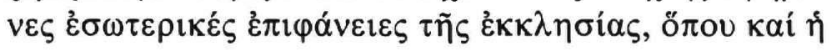

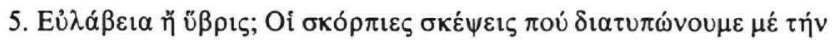

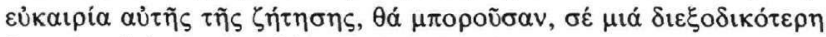

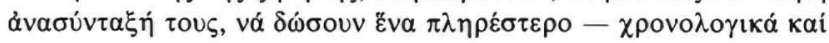

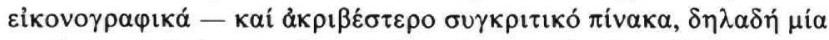

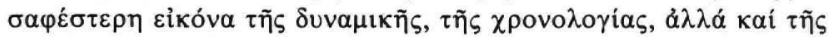

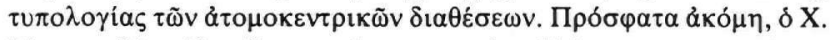

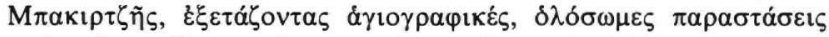

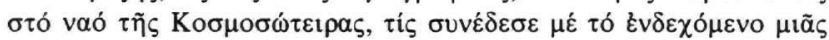

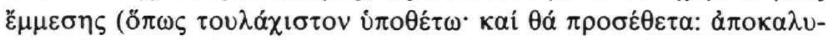

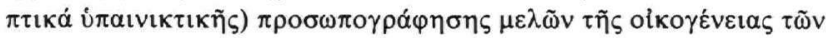

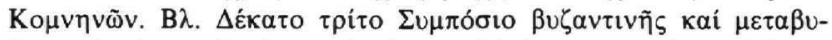

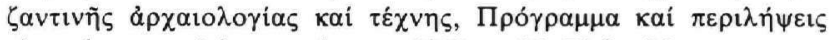

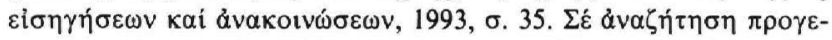

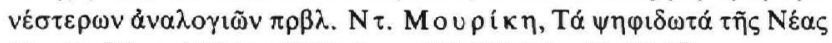
Movĩs Xiov, 'A

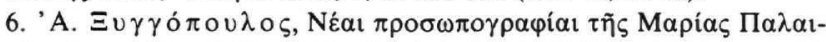

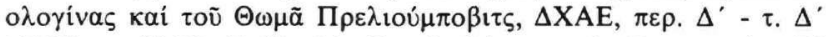

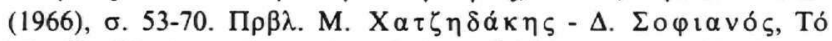

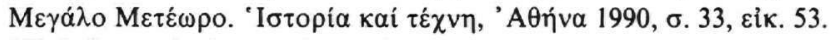

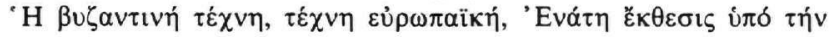

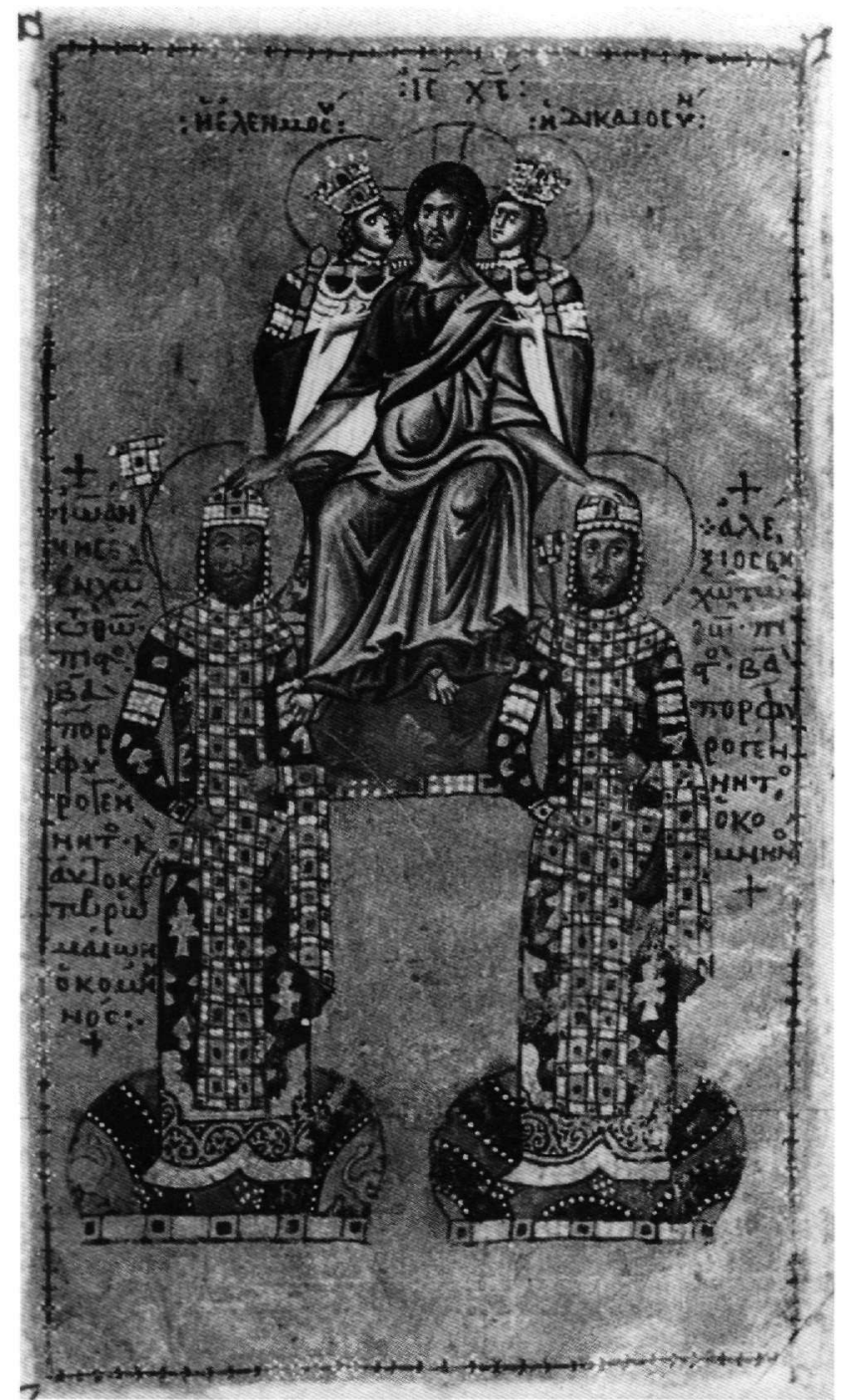

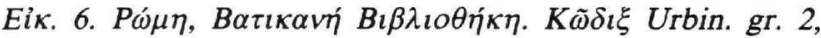
फ. $19 \beta$.

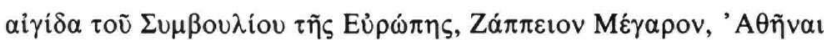

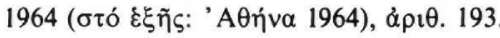

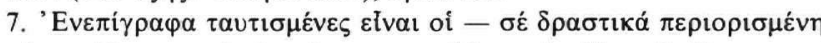

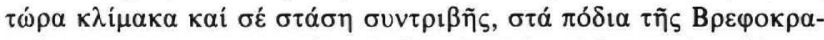

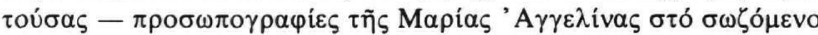

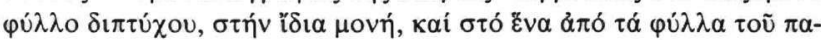

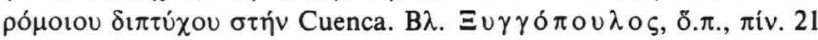

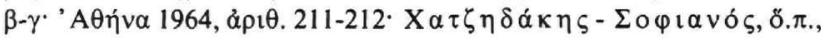
Eǐ. 55.

8. W. Volbach. Elfenbeinarbeiten der Spätantike und des frühen Mittelalters, Mainz $1976^{3}$, d $\rho 1 \theta$. 5. The Metropolitan Museum of Art, New York 1977, Age of Spirituality. Late Antique and Early Chris-

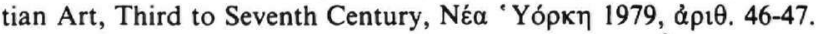
9. A. Grabar, L'Iconoclasme byzantin, П $\alpha \rho i \sigma ı 1984^{2}$, б. 263-264, عiא. 137. K. Weitzmann, The Icon. Holy Images, Sixth to Four-

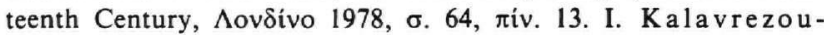

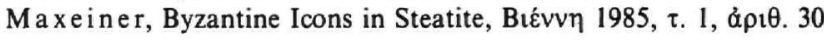

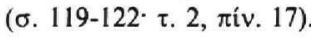




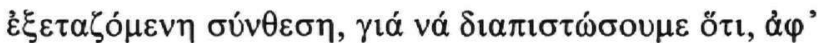

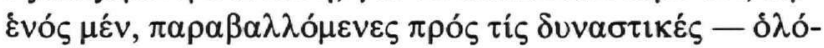

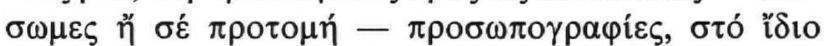

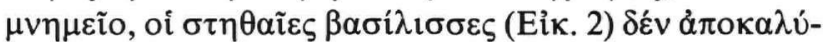

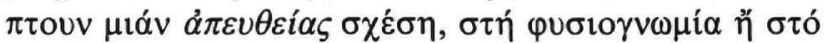

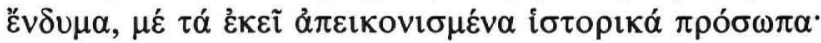

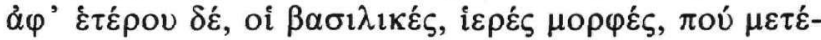

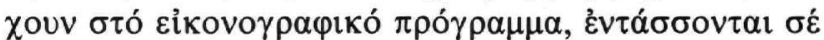

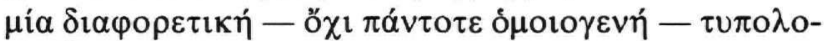
$\gamma i \alpha^{10}$.

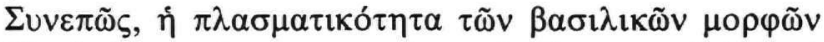

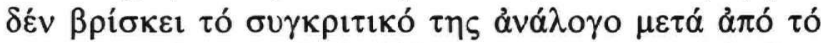

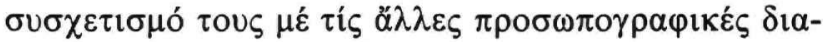

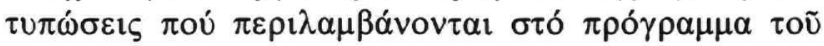

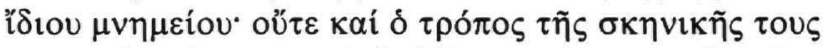

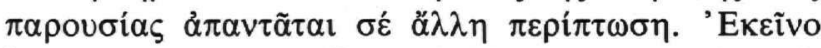

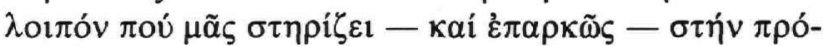

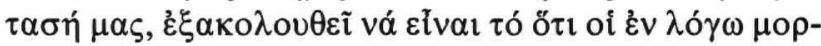

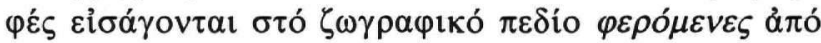

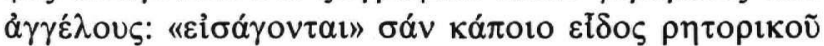

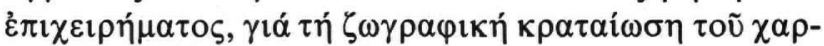

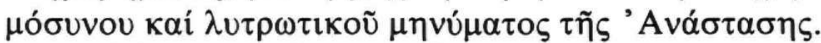

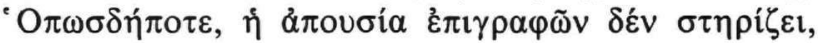

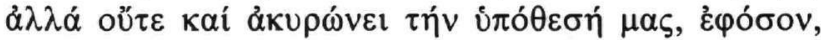

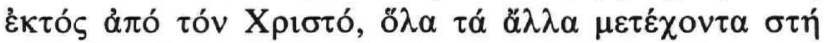

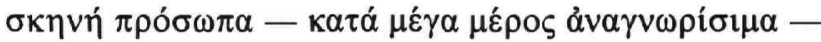

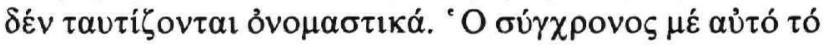

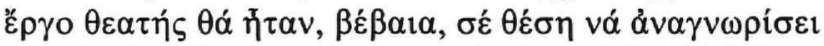

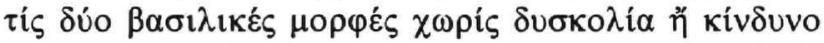

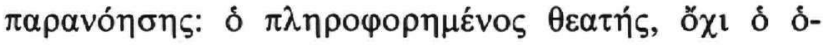

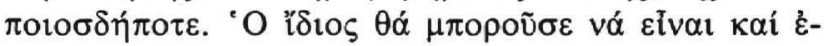

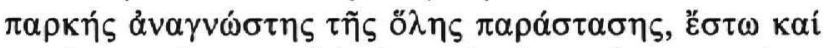

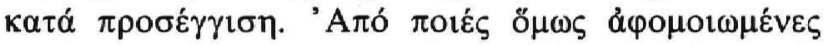

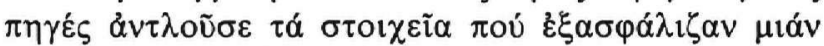

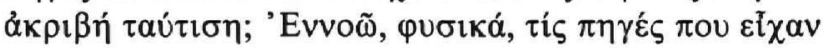

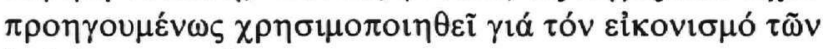
દ́v $\lambda \hat{\gamma} \gamma \omega \pi \rho \circ \sigma \omega ́ \pi \omega v$.

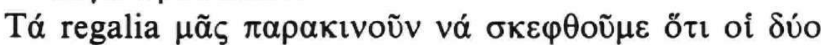

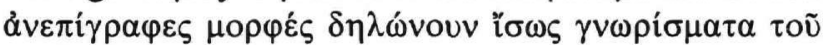

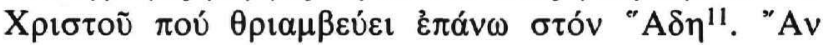

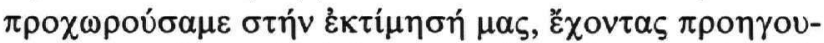

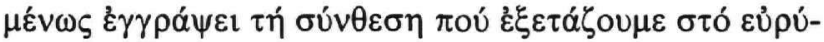

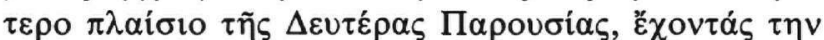

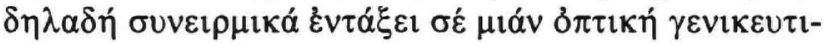

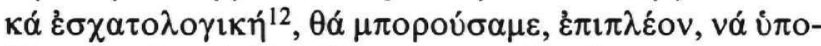

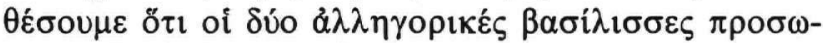

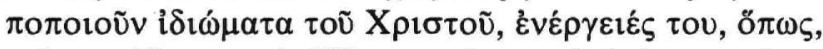

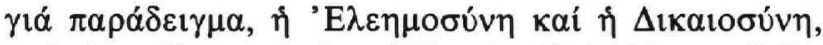

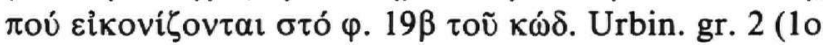

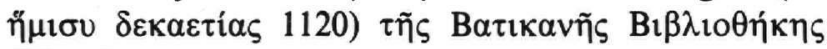
(Eỉk. 6).

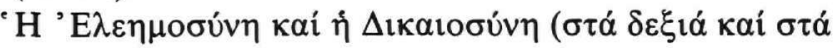

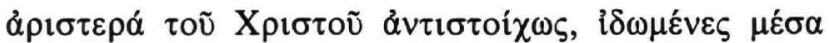

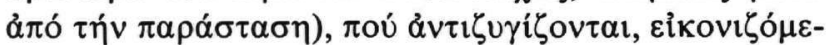

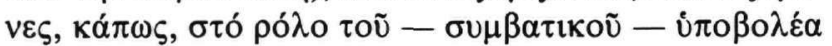

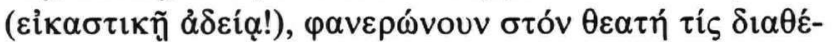

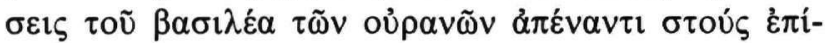

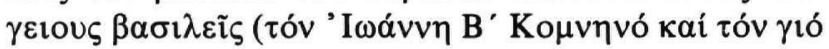

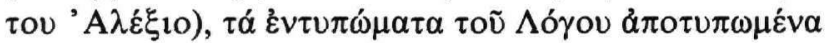

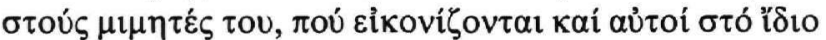

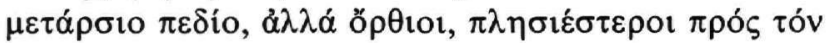

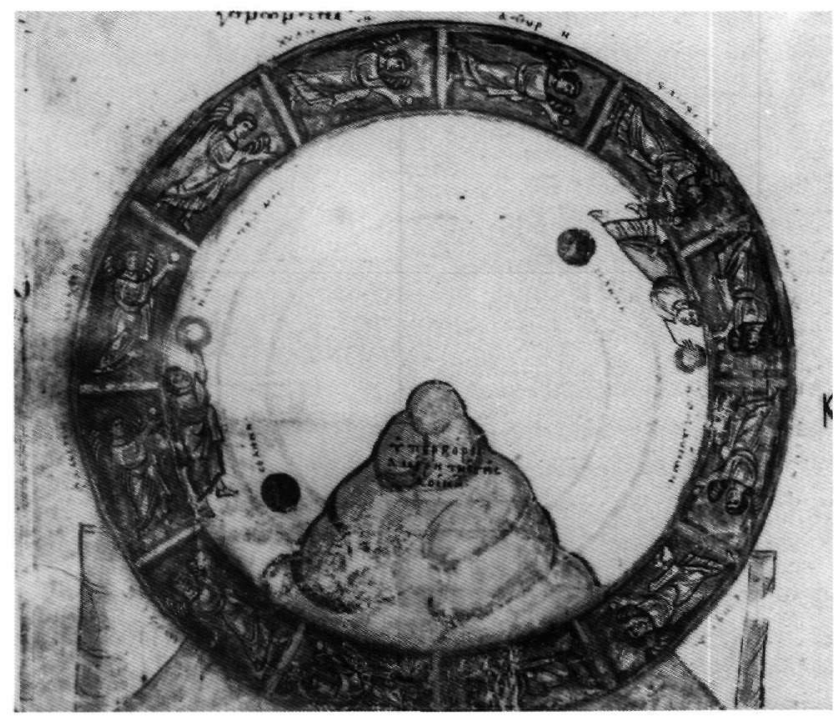

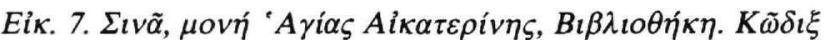
$1186, \varphi .181 \beta$.

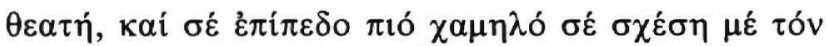

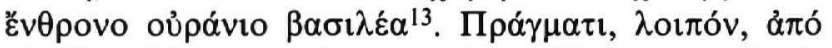

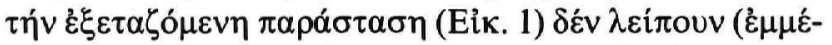

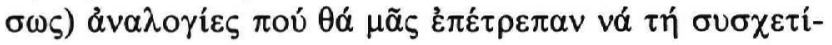

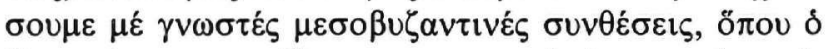

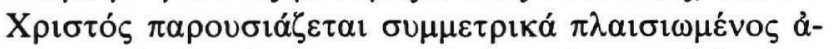

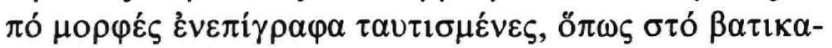

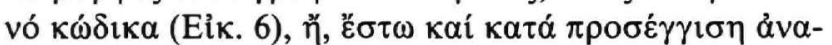

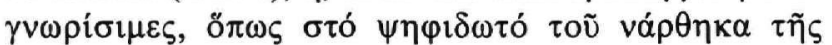

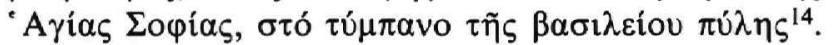

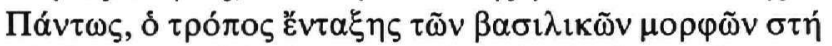

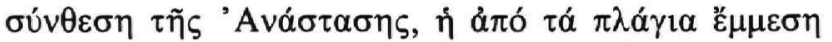

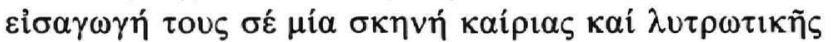

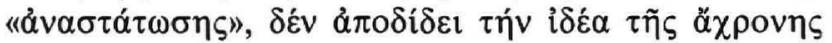

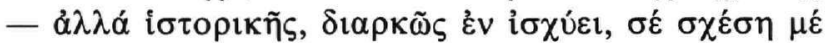

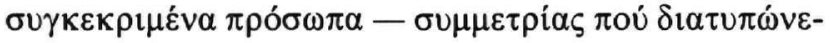

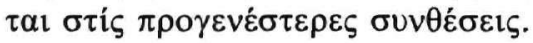

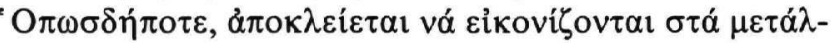

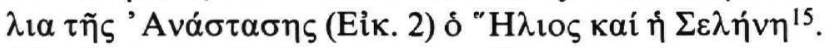

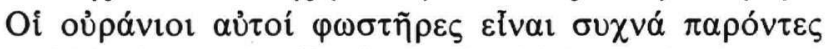

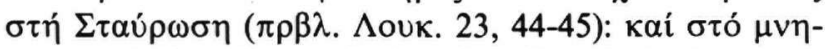

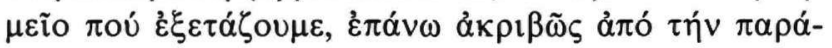




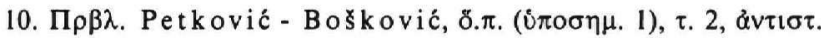

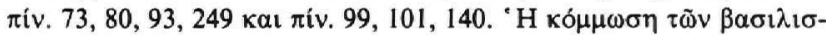

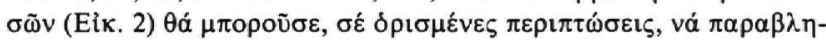

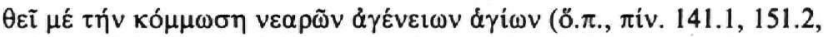

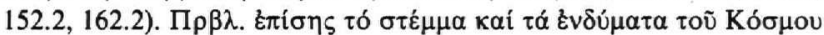

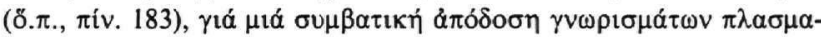

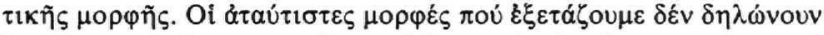

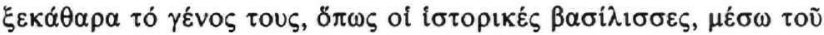

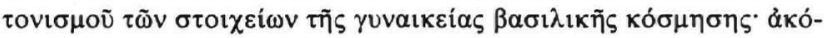

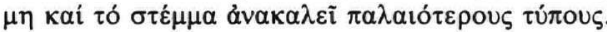

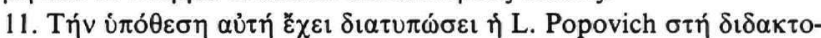

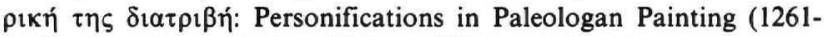
1453), Bryn Mawr College 1963, б. 200-201, 465-466.

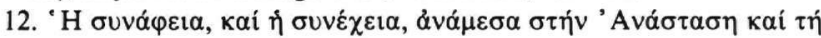

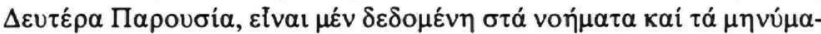

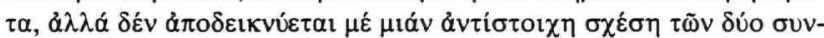

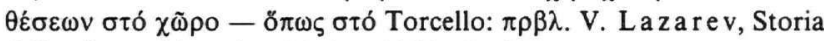
della pittura bizantina, Torino 1967, عik. 370 .

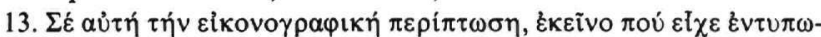

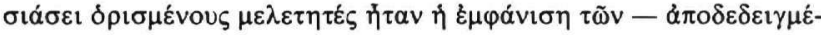

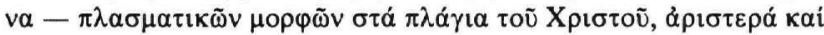

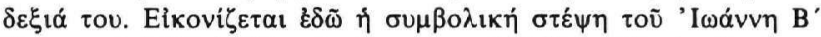

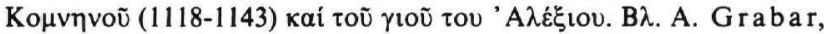
L'empereur dans l'art byzantin, $\Sigma \tau \rho \alpha \sigma \beta$ ○õ $\gamma_{0}$ 1936, $\sigma .119-120$

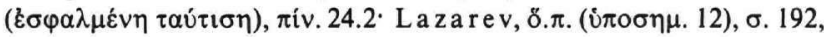

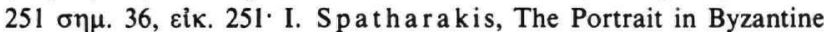

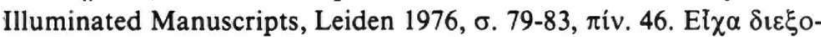

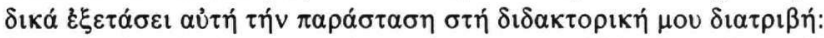
Contribution à l'étude des abstractions personnifiées dans l'art mé-

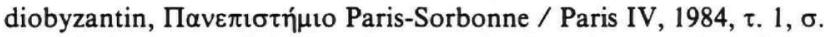

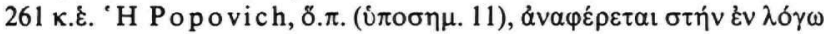

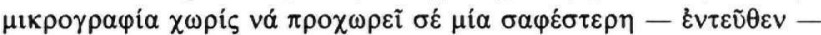

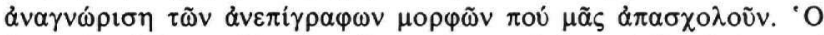

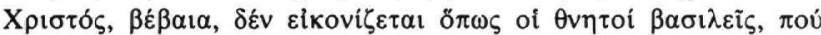

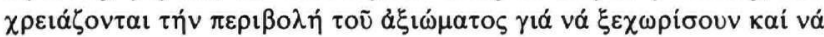

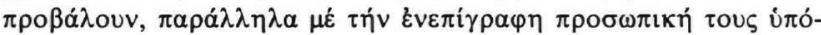

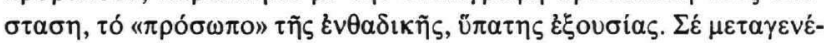

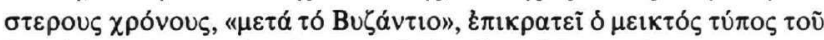

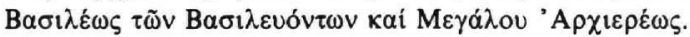

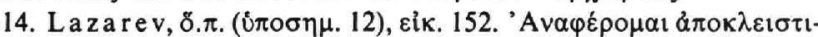

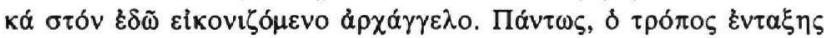

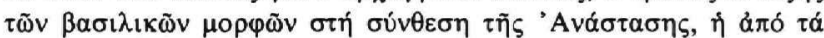

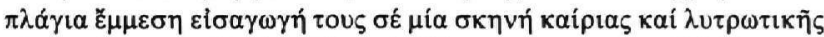

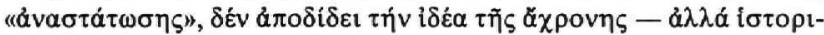

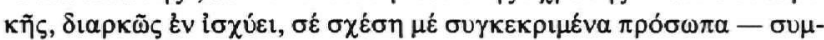

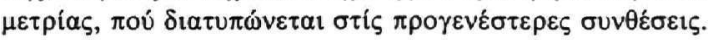

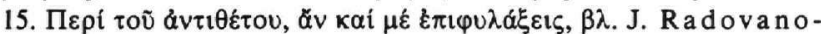
vić, Iconografske zabeleške iz Dečana, Zograf 9 (1978), б. 22-25, 26.

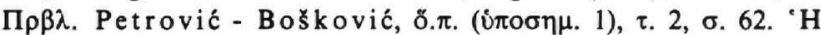

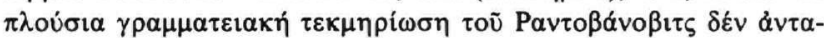

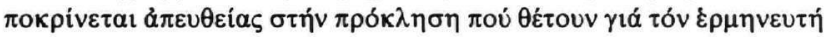

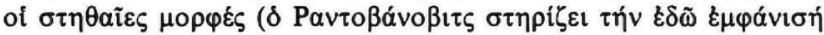

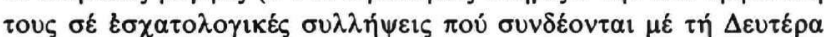

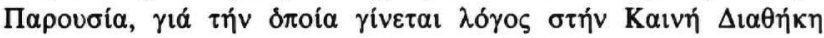

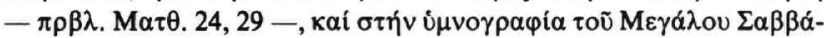

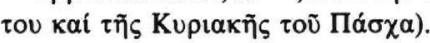

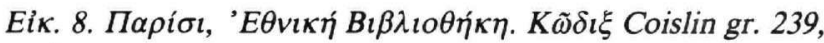
फ. 26.

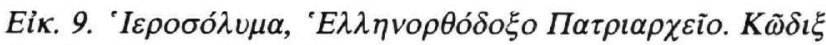

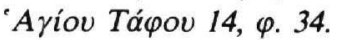
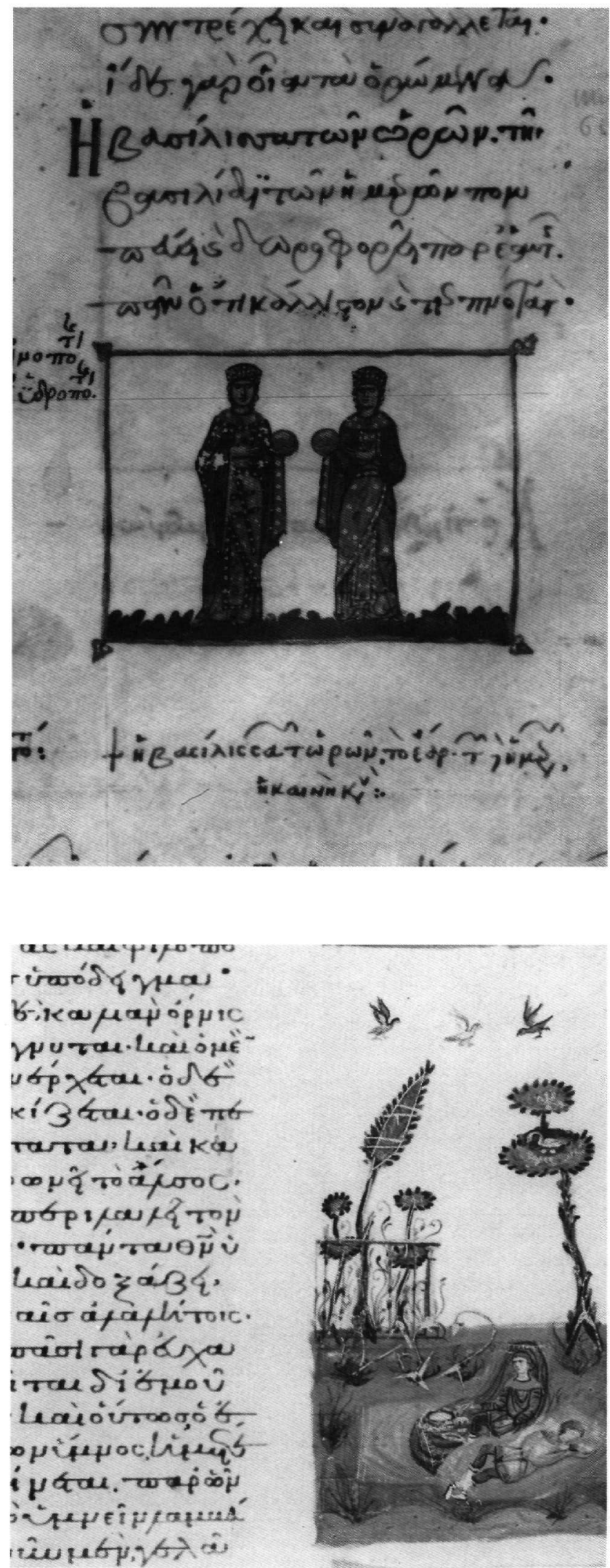


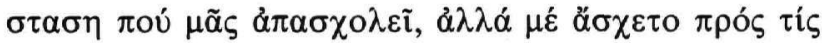

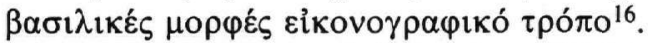

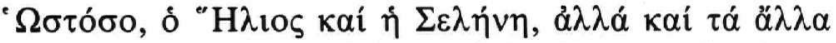

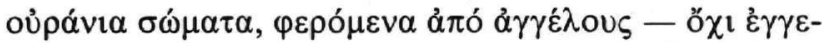

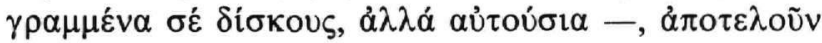

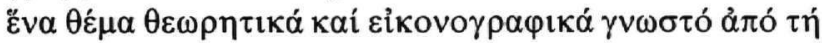

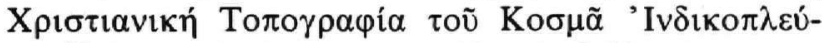

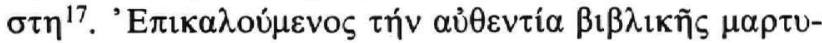

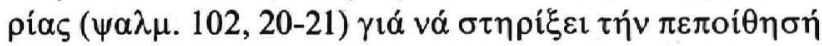

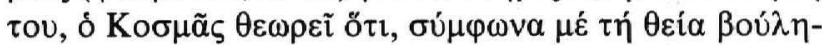

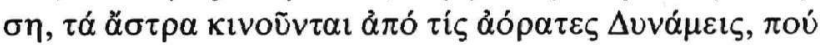

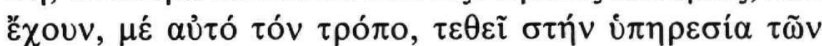

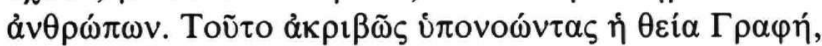

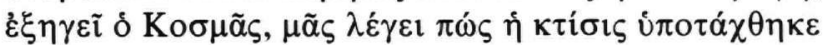

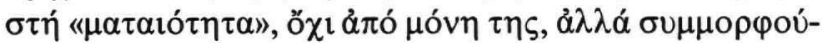

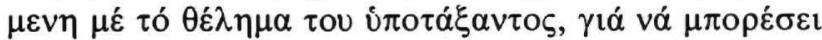

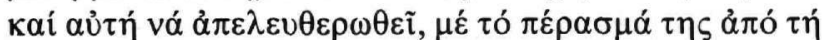

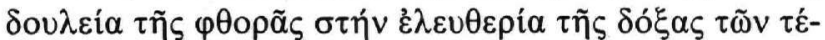

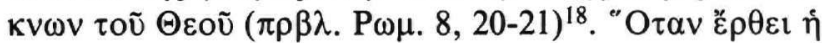

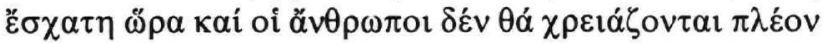

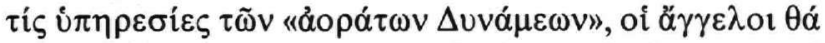

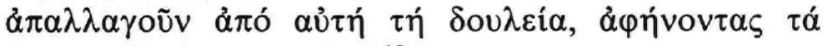

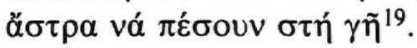

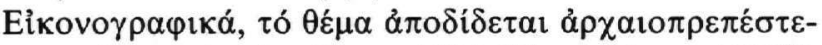

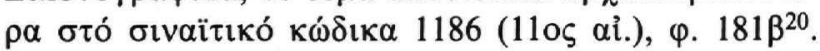

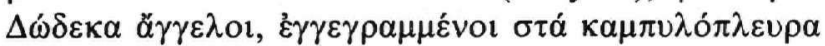

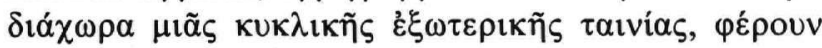

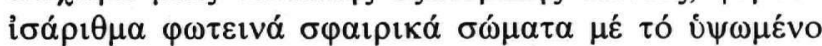

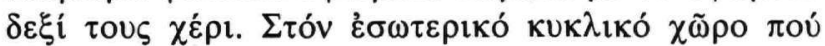

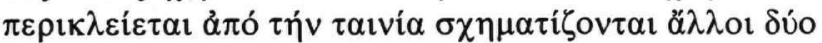

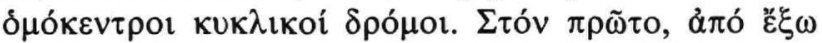

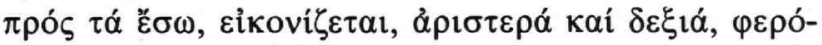

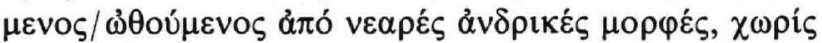

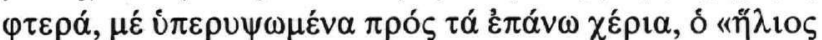

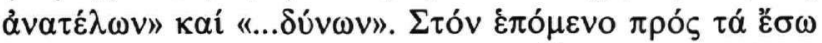

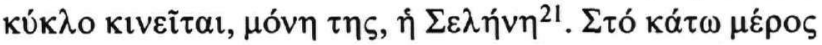

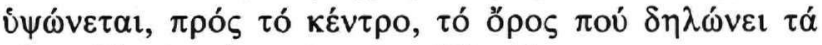

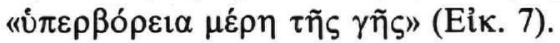

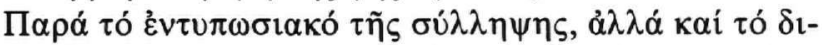

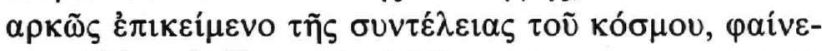

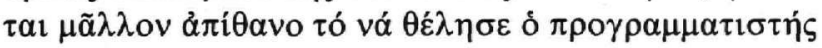

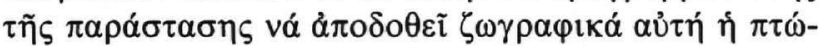

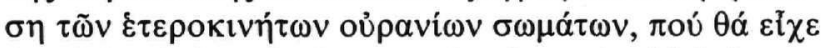

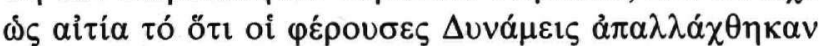

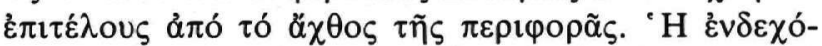

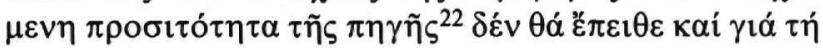

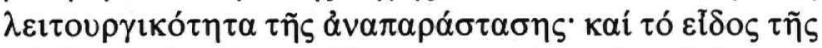

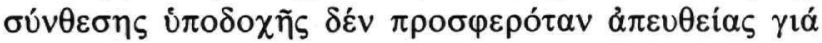

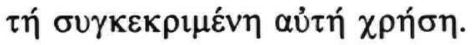

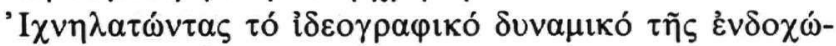

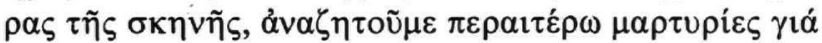

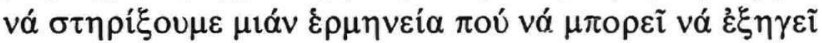

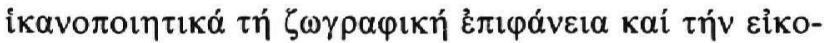
vo $\gamma \rho \alpha \varphi$ เ

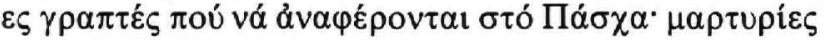

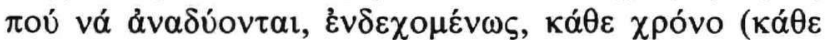

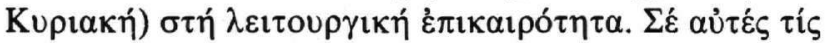

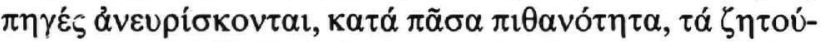

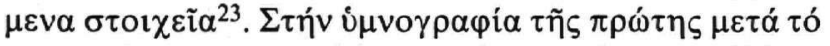

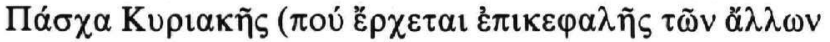

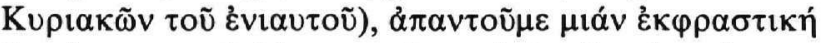

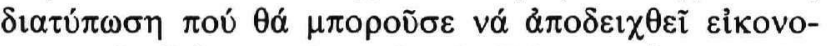

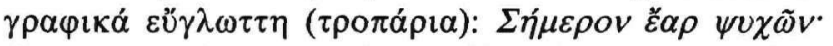

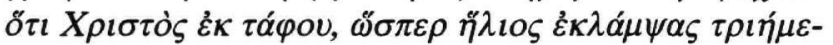
$\rho \circ \varsigma, \tau \dot{\partial} v \zeta o \varphi \varepsilon \rho \dot{o} v \chi \varepsilon \imath \mu \tilde{\omega} v \alpha \dot{\alpha} \pi \tilde{\eta} \lambda \alpha \sigma \varepsilon \tau \tilde{\eta} \varsigma \alpha \dot{\alpha} \mu \alpha \tau i \dot{\alpha} \alpha \tilde{\eta} \mu \tilde{\omega} v$ (...) / 'H $\beta \alpha \sigma i \lambda i \varsigma \tau \tilde{\omega} v \omega \rho \tilde{\omega} v, \tau \tilde{\eta} \lambda \alpha \mu \pi \rho \circ \varphi o ́ \rho \omega \eta \dot{\eta} \mu \varepsilon \rho \alpha$, $\tilde{\eta} \mu \varepsilon \rho \tilde{\omega} v \tau \varepsilon \beta \alpha \sigma \imath \lambda i \delta$ เ

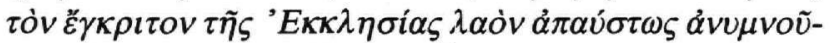

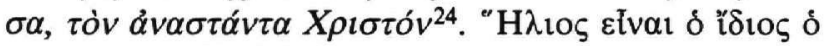

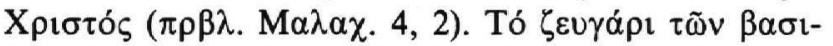

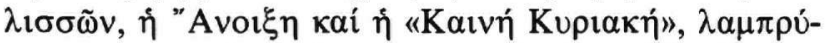

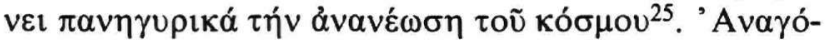

16. S. Djurić, The Representations of Sun and Moon at Dečani,

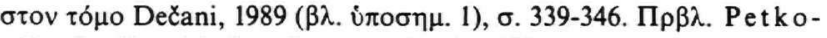

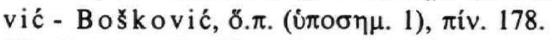

17. Cosmas Indicopleustès, Topographie chrétienne, $\tau$. 3, П $\alpha$ -

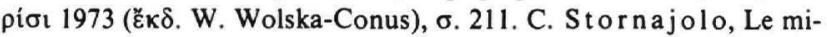
niature della Topografia Cristiana di Cosma Indicopleuste, Mı $\lambda$ ávo 1908. K. Weitzmann - G. Galavaris, The Monastery of Saint Catherine at Mount Sinai. The Illuminated Greek Manuscripts, I: From the Ninth to the Twelfth Century, Princeton 1990, б. 7, 52-65.

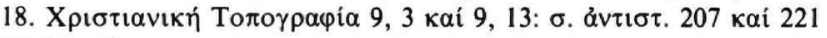
Wolska-Conus.

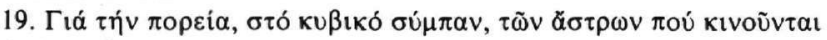

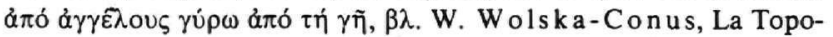
graphie chrétienne de Cosmas Indicopleustès. Théologie et science au VIe siècle, Парí⿴囗 1962, б. 168, 180-181.

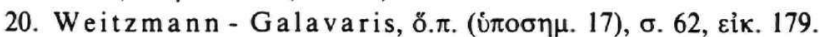

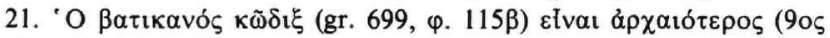

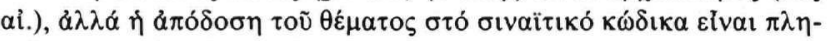

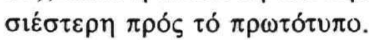

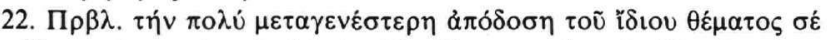

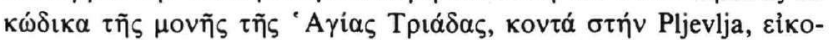

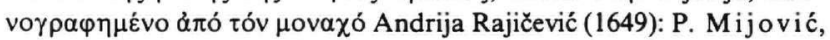

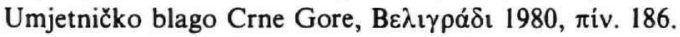

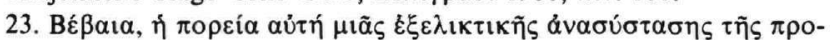

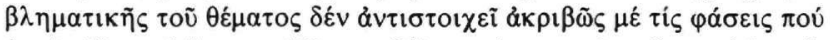

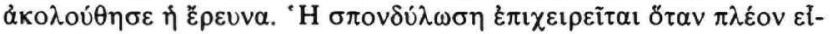

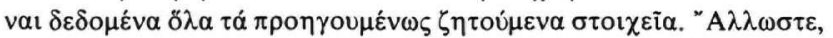

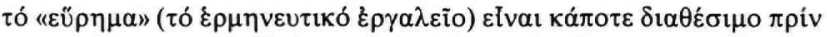

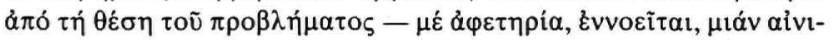

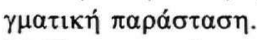

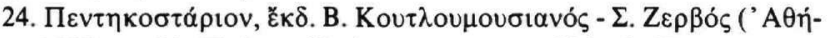

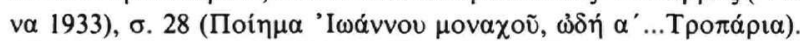

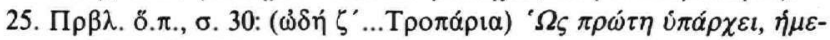

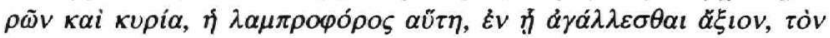

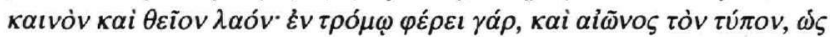

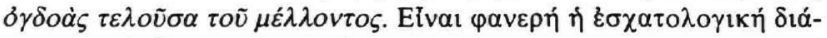

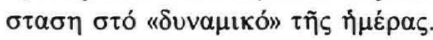




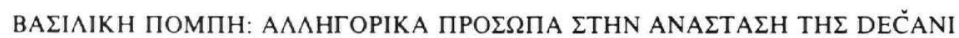

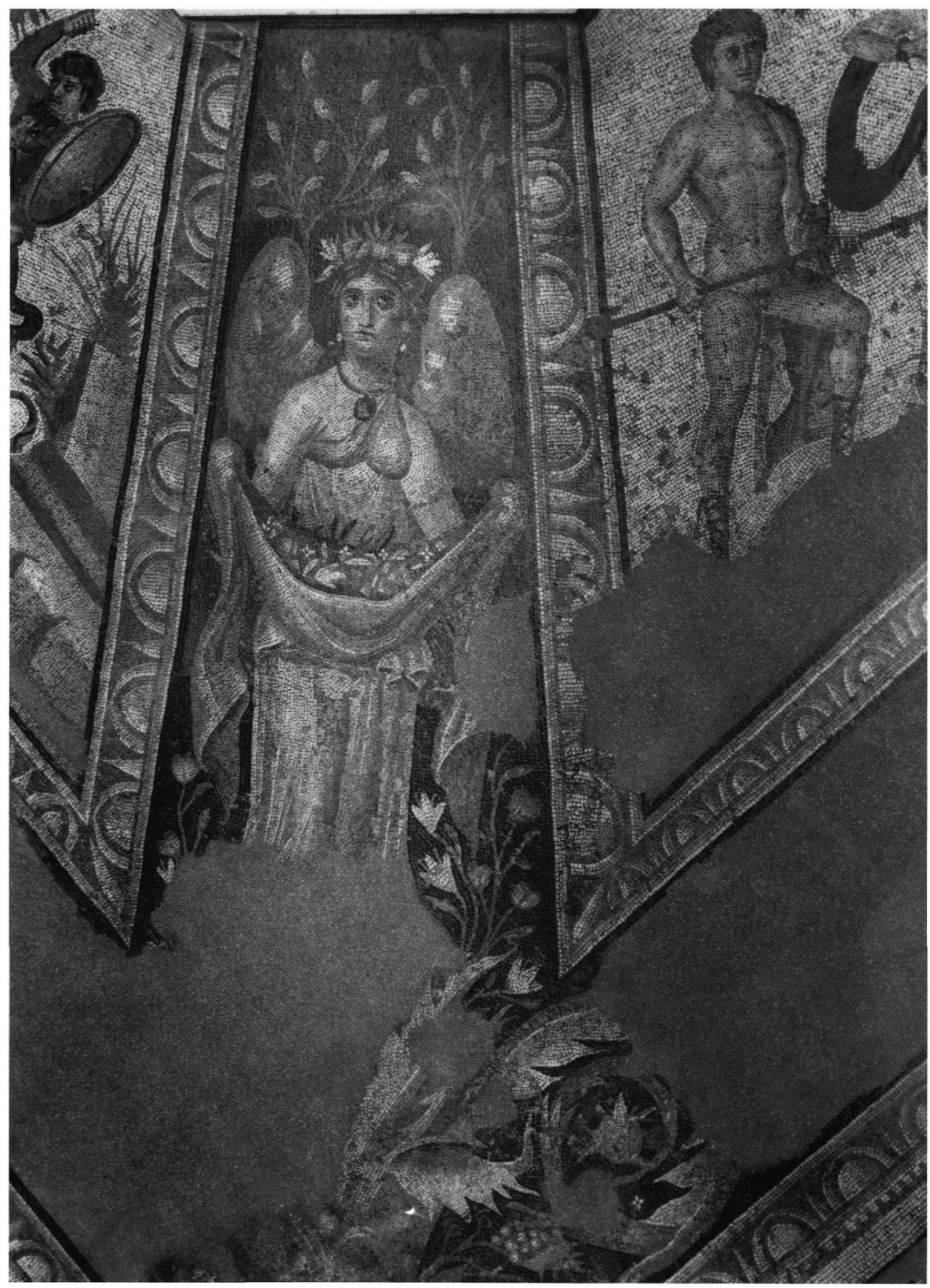

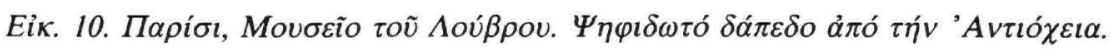




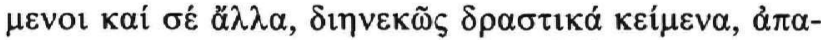

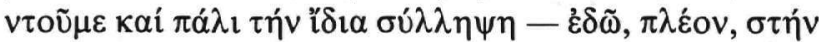

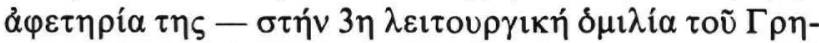

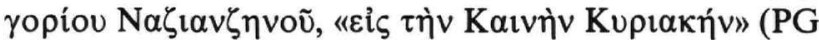

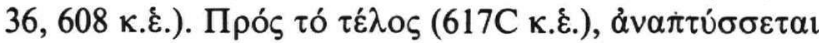

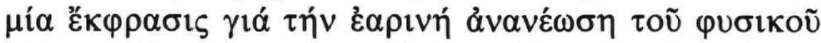

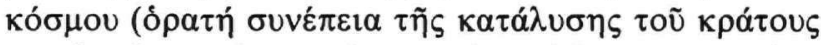

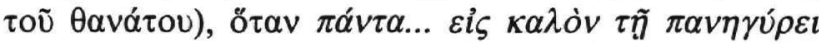

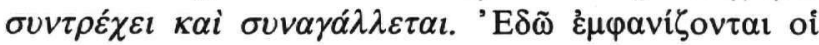

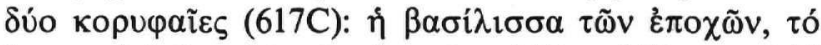

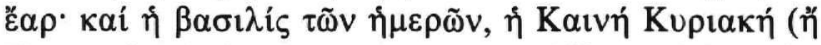

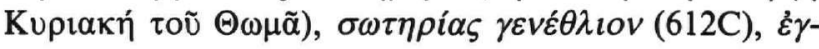

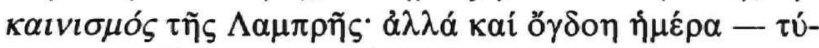

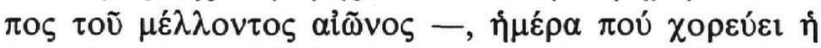

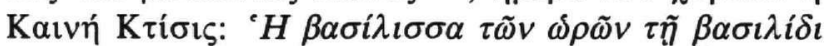

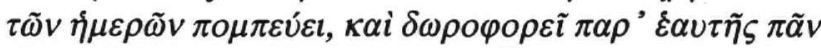

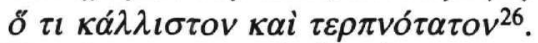

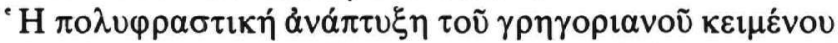

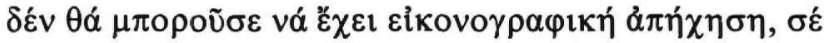

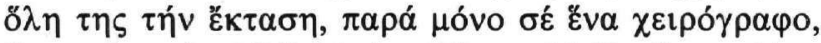

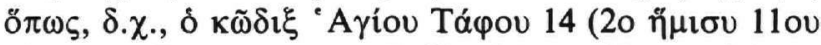

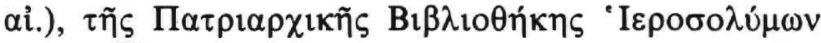

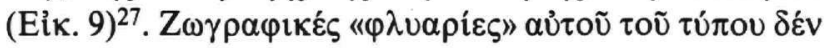

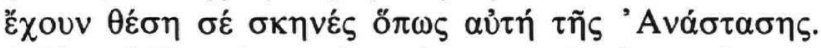

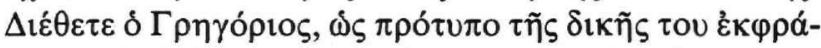

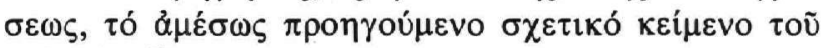

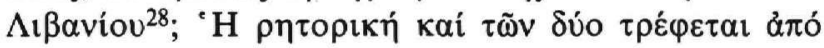

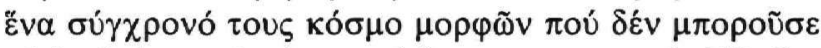

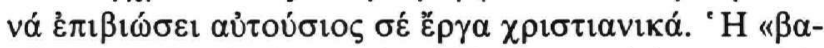

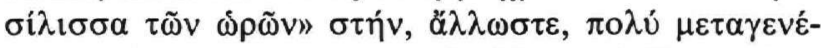

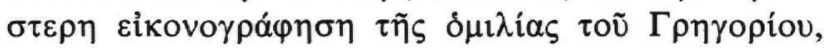

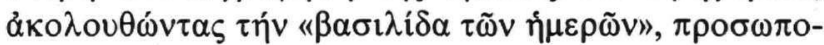

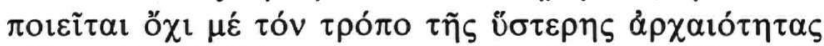

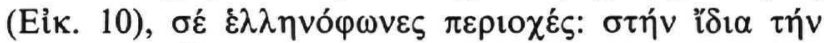

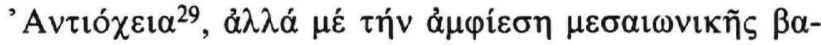

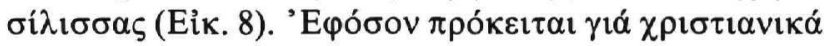

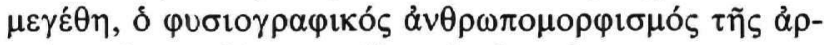

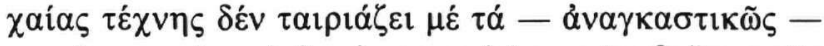

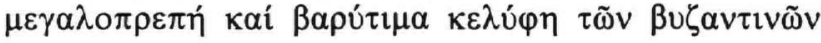

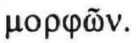

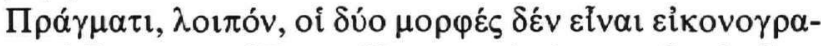

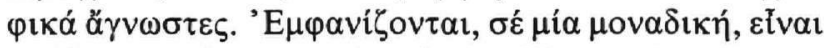

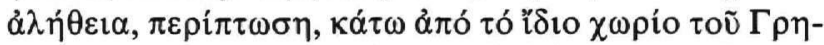

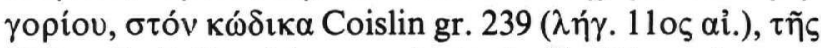

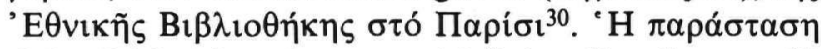

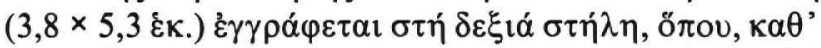

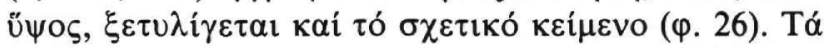

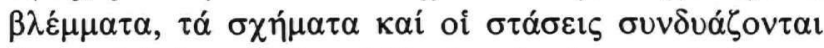

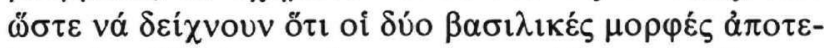

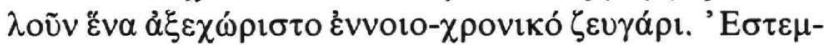

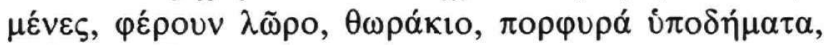

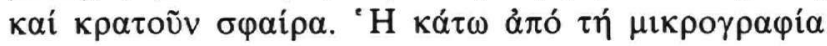

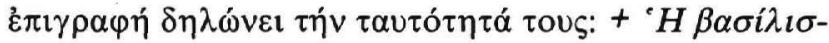
$\sigma \alpha \tau(\tilde{\omega} v) \omega \rho \tilde{\omega} v \tau \dot{o} \varepsilon \tilde{\varepsilon} \alpha \cdot \tau(\tilde{\omega} v)(\delta \dot{\varepsilon}) \dot{\eta} \mu \varepsilon \rho(\tilde{\omega} v) / \dot{\eta} K \alpha \imath v \dot{\eta}$

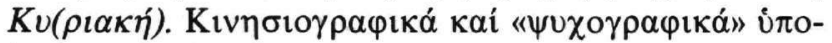

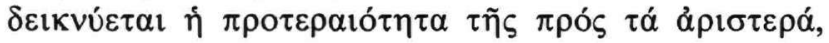

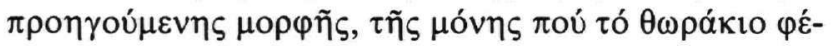

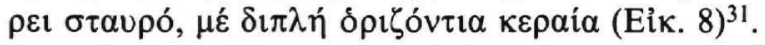

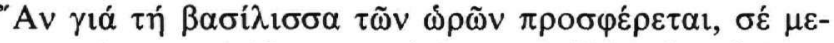

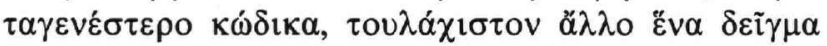

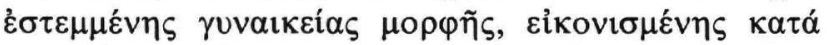

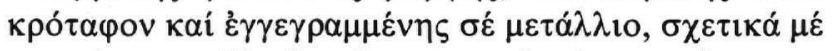

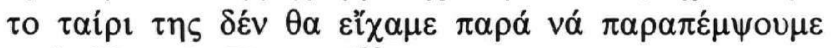

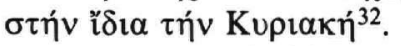

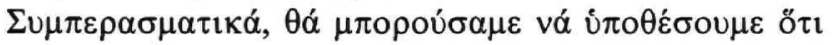

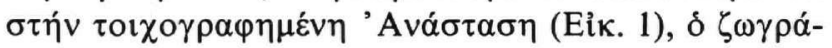

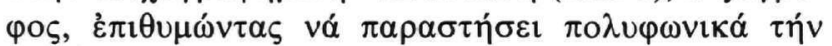

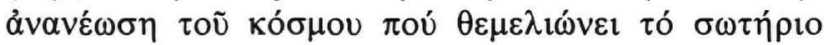

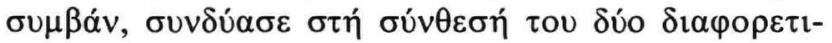

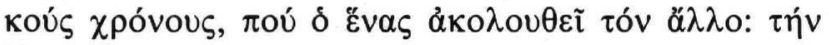

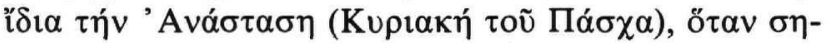

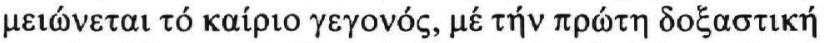

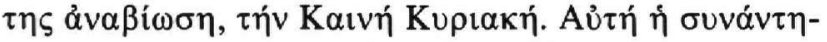

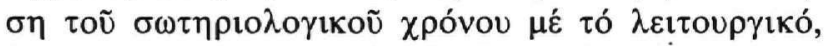

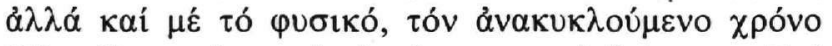

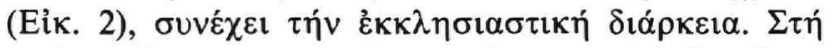

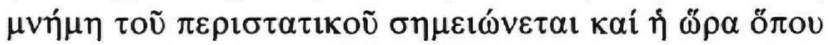

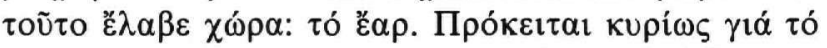

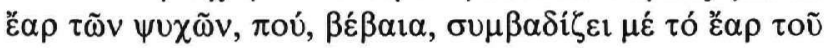

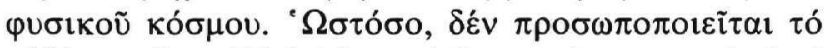

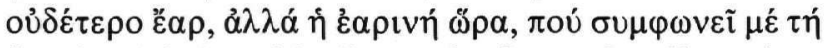

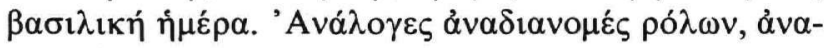

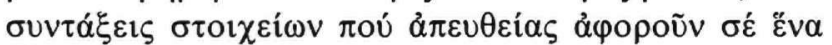

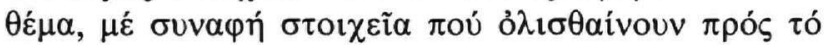

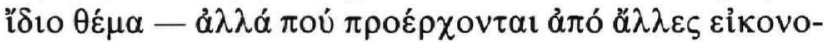

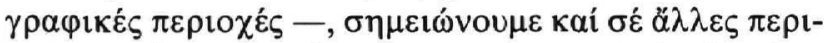

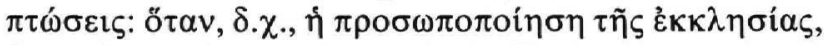

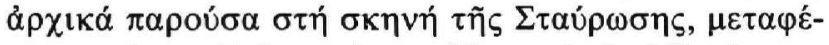

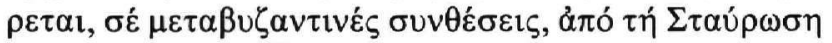
$\sigma \tau \eta \dot{v}{ }^{\prime} A v \alpha ́ \sigma \tau \alpha \sigma \eta^{33}$.

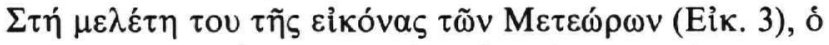

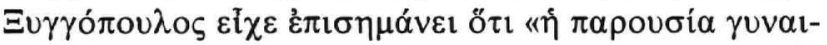

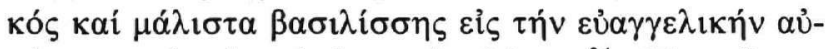

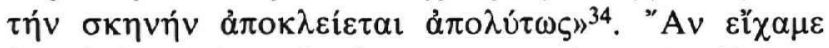

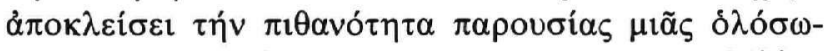

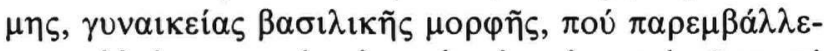

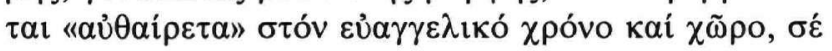
$\mu i \alpha$

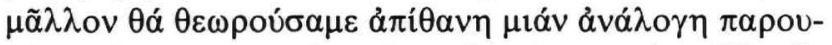

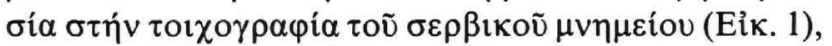

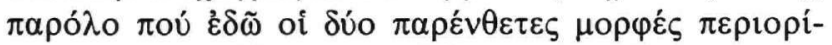

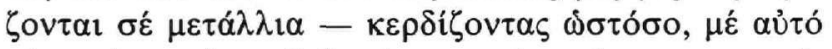

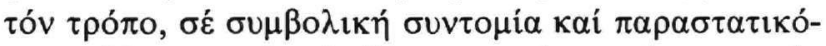

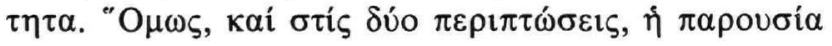




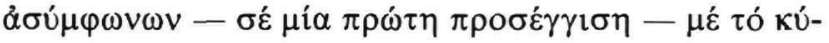

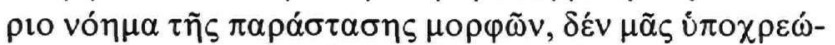

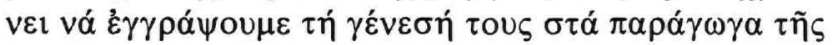

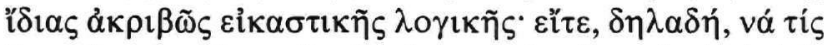

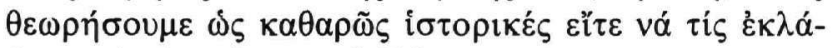

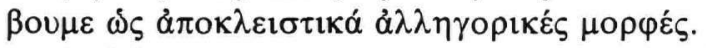

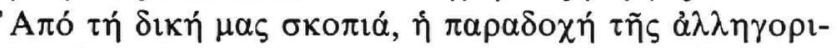

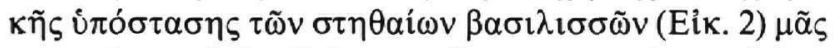

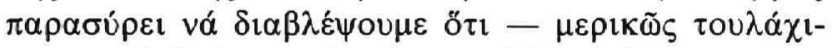

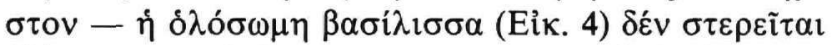

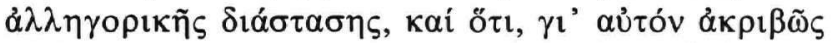

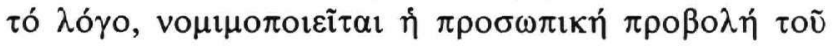

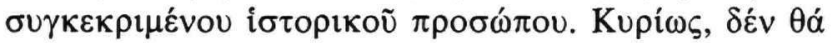

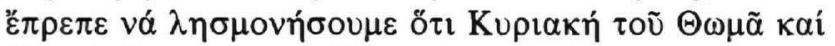

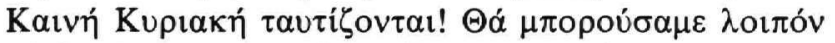

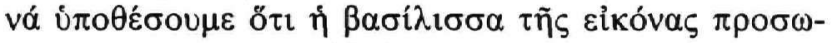

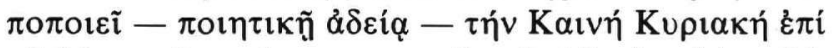

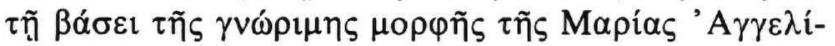

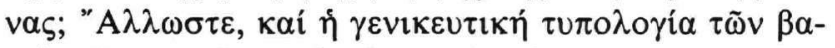

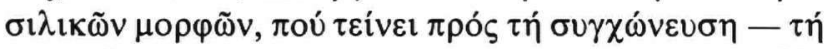

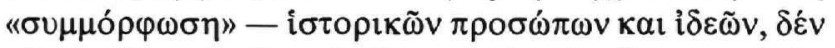

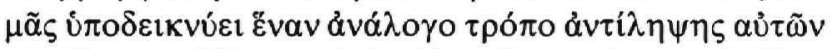

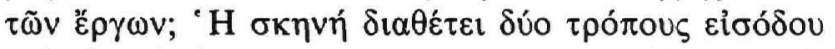

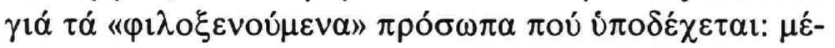

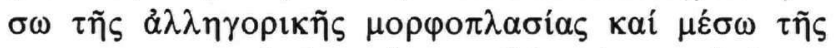

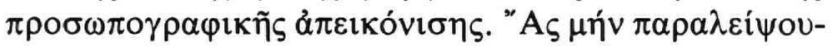

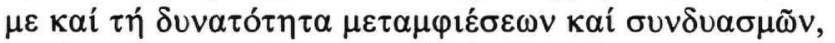

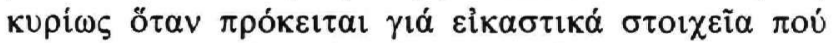

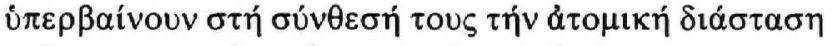

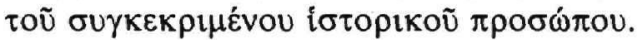

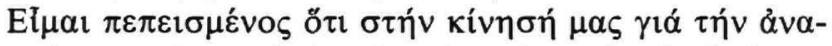

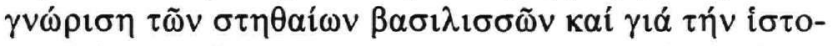

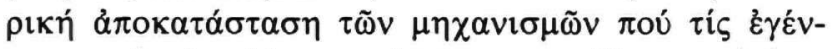

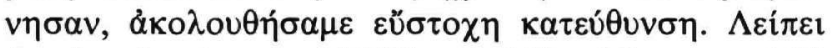

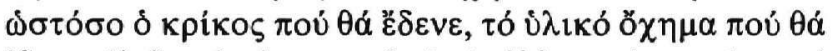

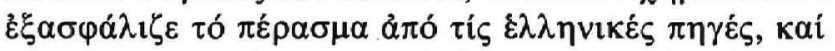

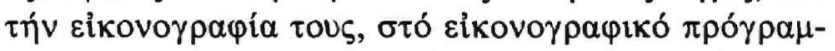

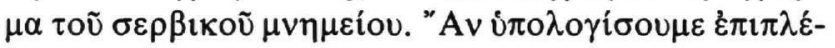

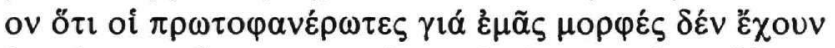

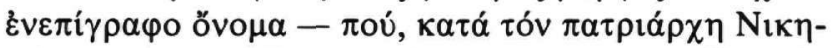

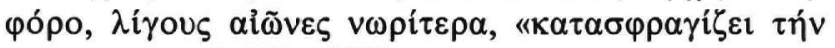

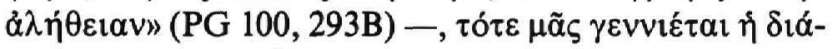

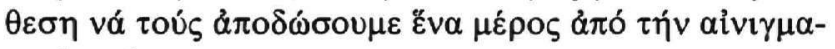

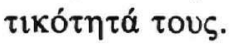

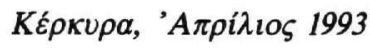

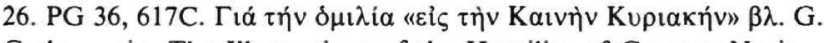
$\mathrm{G} a$ lavaris, The Illustrations of the Homilies of Gregory Nazian-

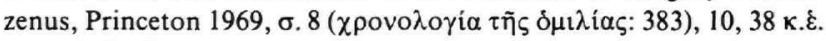

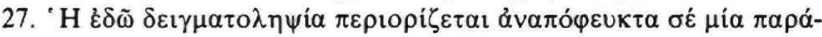

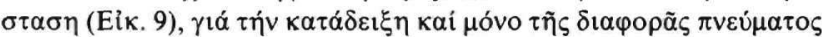

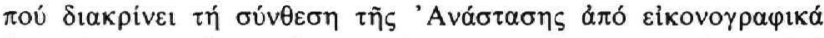

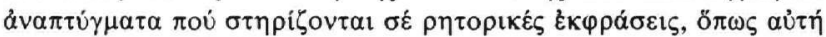

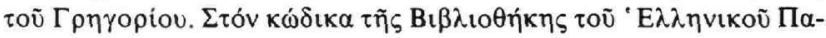

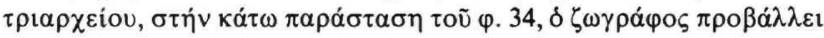

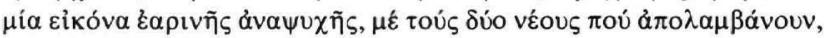

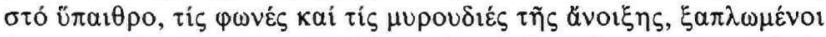

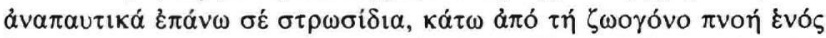

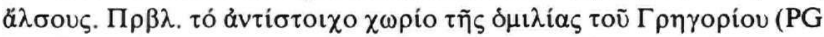

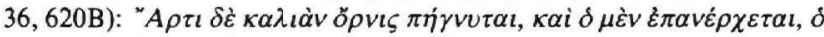

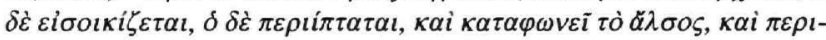

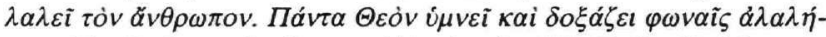

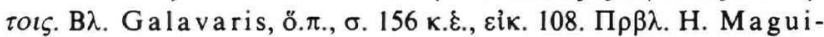
re, Art and Eloquence in Byzantium, Princeton 1981, o. 42-44, Eỉ . 30.

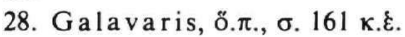

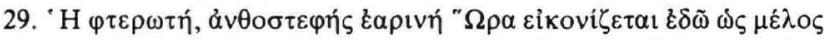

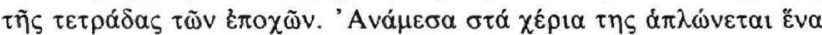

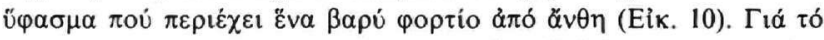

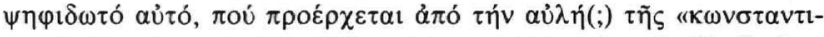

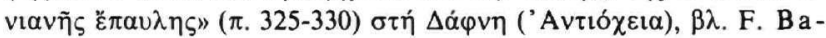
ratte, Catalogue des mosaïques romaines et paléochrétiennes du

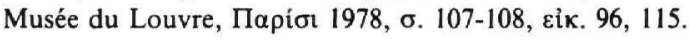

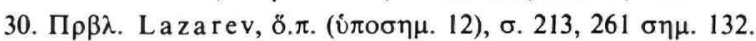

31. H. Omont, Miniatures des plus anciens manuscrits grecs de la Bibliothèque nationale, du VIe au XIVe siècle, Пapía 1929, б. 55,

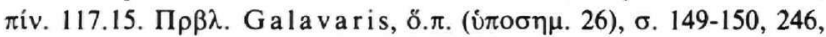
عǐk. 197.

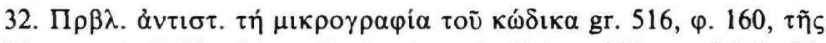

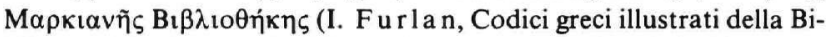

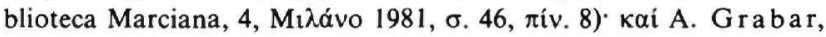
L'iconographie du dimanche, principalement à Byzance, Lex orandi,

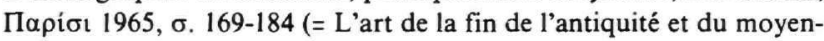

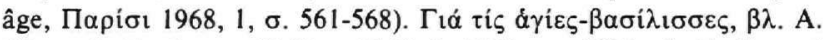

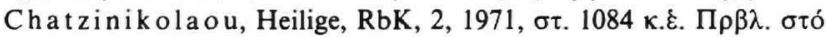

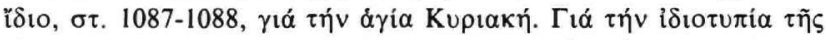

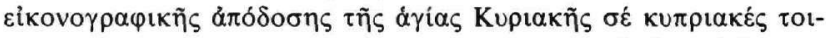

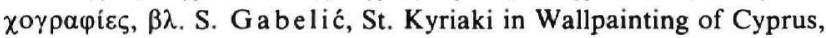

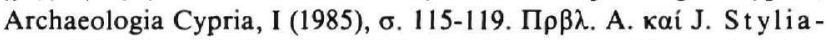
nou, The Painted Churches of Cyprus. Treasures of Byzantine Art,

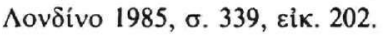

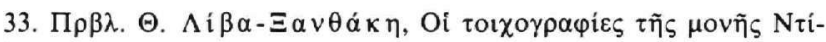

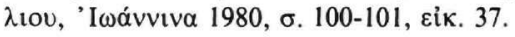

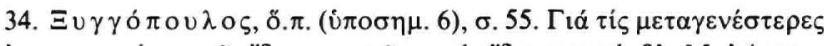

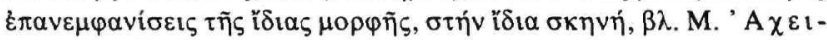

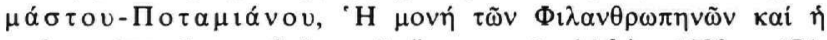

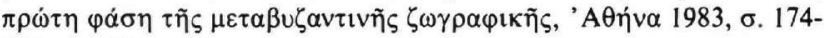
175 , عik. $78 \alpha$. 


\section{Elias Antonopoulos}

\section{CORTÈGE ROYAL: PERSONNAGES ALLÉGORIQUES DANS L'ANASTASIS DE DEČANI}

$\mathrm{O}_{\mathrm{n}}$ avait précédemment supposé que dans les deux médaillons que des anges introduisent dans la composition de l'Anastasis, à Dečani (ca. m. XIVe s.), sont figurés: le Soleil, ou, ensemble, les deux luminaires du ciel (le Soleil et la Lune), ou, enfin, des aspects glorieux du Christ ressuscité - ce qui pouvait paraître plus confortablement vraisemblable (Fig. 1-2). Il s'agit en effet de jeunes personnages féminins, frontalement figurés, en buste, et portant la parure royale. A la lumière de l'identité donnée de deux reines: "la reine des jours" et "la reine de saisons" (Fig. 8), qui apparaissent, vues en pied, en illustration d'un passage de l'homélie sur le Nouveau Dimanche (PG 36, col. 608 sq.) de Grégoire de Nazianze, dans un codex (fin XIe s.) de la Bibliothèque nationale de Paris (Coislin gr. 239, f. 26), une nouvelle proposition, plus précise que les précédentes, soutient que les figures anépigraphes de Dečani (Fig. 2) personnifient, justement, le reine des jours (premier dimanche après Pâques), et la reine des saisons (le printemps). Or ce dimanche est également le jour où on commémore l'incrédulité de Thomas. Dans une icône (fin XIVe s.) figurant cet événement, conservée au monastère de la Transfiguration (Météôra), on peut voir une reine, non identifiée, parmi les apôtres (Fig. 3-4). Sans contester une identification antérieure, celle d'André Xyngopoulos pensant qu'il s'agit de Marie Paléologine, épouse du despote de Jannina Thomas Preljubović, il est ici suggéré que cette reine, tout en figurant (probablement) un personnage historique précis, revêt en même temps des allures allégoriques - à cause, justement, de son apparition dans un tel contexte. Serait-ce son alibi pour y être introduite? 